\title{
Double beta decay: present status
}

\author{
A.S. Barabash ${ }^{1}$ \\ ${ }^{1}$ Institute of Theoretical and Experimental Physics
}

\begin{abstract}
The present status of double beta decay experiments (including the search for $2 \beta^{+}$, $\mathrm{EC} \beta^{+}$and ECEC processes) are reviewed. The results of the most sensitive experiments are discussed. Average and recommended half-life values for two-neutrino double beta decay are presented. Conservative upper limits on effective Majorana neutrino mass and the coupling constant of the Majoron to the neutrino are established as $\left\langle m_{\nu}\right\rangle<0.75 \mathrm{eV}$ and $\left\langle g_{e e}\right\rangle<1.9 \cdot 10^{-4}$, respectively. Proposals for future double beta decay experiments with a sensitivity for the $\left\langle m_{\nu}\right\rangle$ at the level of (0.01-0.1) $\mathrm{eV}$ are considered.
\end{abstract}

\section{INTRODUCTION}

The current interest in neutrinoless double beta decay, $0 \nu \beta \beta$ decay, is that the existence of this process is closely related to the following fundamental aspects of particle physics [1-3]: (i) lepton-number nonconservation, (ii) the presence of a neutrino mass and its origin, (iii) the existence of right-handed currents in electroweak interactions, (iv) the existence of the Majoron, (v) the structure of the Higgs sector, (vi) supersymmetry, (vii) the existence of leptoquarks, (viii) the existence of a heavy sterile neutrino, and (ix) the existence of a composite neutrino.

All of these issues are beyond the standard model of electroweak interaction, therefore the detection of $0 \nu \beta \beta$ decay would imply the discovery of new physics. Of course, interest in this process is caused primarily by the problem of a neutrino mass. If $0 \nu \beta \beta$ decay is discovered, then according to current thinking, this will automatically mean that the rest mass of at least one neutrino flavor is nonzero and is of Majorana origin.

Interest in neutrinoless double-beta decay has seen a significant renewal in recent years after evidence for neutrino oscillations was obtained from the results of atmospheric, solar, re-

E-mail: barabash@itep.ru 
actor and accelerator neutrino experiments (see, for example, the discussions in [4-6]). These results are impressive proof that neutrinos have a non-zero mass. However, the experiments studying neutrino oscillations are not sensitive to the nature of the neutrino mass (Dirac or Majorana) and provide no information on the absolute scale of the neutrino masses, since such experiments are sensitive only to the difference of the masses, $\Delta m^{2}$. The detection and study of $0 \nu \beta \beta$ decay may clarify the following problems of neutrino physics (see discussions in [7-9]): (i) lepton number non-conservation, (ii) neutrino nature: whether the neutrino is a Dirac or a Majorana particle, (iii) absolute neutrino mass scale (a measurement or a limit on $m_{1}$ ), (iv) the type of neutrino mass hierarchy (normal, inverted, or quasidegenerate), (v) $\mathrm{CP}$ violation in the lepton sector (measurement of the Majorana CP-violating phases).

Let us consider three main modes of $2 \beta$ decay ${ }^{1}$ :

$$
\begin{gathered}
(A, Z) \rightarrow(A, Z+2)+2 e^{-}+2 \tilde{\nu} \\
(A, Z) \rightarrow(A, Z+2)+2 e^{-} \\
(A, Z) \rightarrow(A, Z+2)+2 e^{-}+\chi^{0}\left(+\chi^{0}\right)
\end{gathered}
$$

The $2 \nu \beta \beta$ decay (process (1)) is a second-order process, which is not forbidden by any conservation law. The detection of this process provides the experimental determination of the nuclear matrix elements (NME) involved in the double beta decay processes. This leads to the development of theoretical schemes for NME calculations both in connection with the $2 \nu \beta \beta$ decays as well as the $0 \nu \beta \beta$ decays [10-13]. Moreover, the study can yield a careful investigation of the time dependence of the coupling constant for weak interactions [14-16].

Recently, it has been pointed out that the $2 \nu \beta \beta$ decay allows one to investigate particle properties, in particular whether the Pauli exclusion principle is violated for neutrinos and thus neutrinos partially obey Bose-Einstein statistics [17, 18].

\footnotetext{
${ }^{1}$ The decay modes also include $(\mathrm{A}, \mathrm{Z})$ - $(\mathrm{A}, \mathrm{Z}-2)$ processes via (i) the emission of two positrons $\left(2 \beta^{+}\right.$ processes), (ii) the emission of one positron accompanied by electron capture (EC $\beta^{+}$processes), and (iii) the capture of two orbital electrons (ECEC). For the sake of simplicity, we will consider $2 \beta^{-}$decay. In each case where it will be desirable to invoke $2 \beta^{+}, \mathrm{EC} \beta^{+}$, or ECEC processes, this will be indicated specifically.
} 
The $0 \nu \beta \beta$ decay (process $(2))$ violates the law of lepton-number conservation $(\Delta L=2)$ and requires that the Majorana neutrino has a nonzero rest mass or that an admixture of right-handed currents be present in weak interaction. Also, this process is possible in some supersymmetric models, where $0 \nu \beta \beta$ decay is initiated by the exchange of supersymmetric particles. This decay also arises in models featuring an extended Higgs sector within electroweak-interaction theory and in some other cases [1].

The $0 \nu \chi^{0} \beta \beta$ decay (process $\left.(3)\right)$ requires the existence of a Majoron. It is a massless Goldstone boson that arises due to a global breakdown of (B -L) symmetry, where B and L are, respectively, the baryon and the lepton number. The Majoron, if it exists, could play a significant role in the history of the early Universe and in the evolution of stars. The model of a triplet Majoron [19] was disproved in 1989 by the data on the decay width of the $Z^{0}$ boson that were obtained at the LEP accelerator (CERN, Switzerland) [20]. Despite this, some new models were proposed [21, 22], where $0 \nu \chi^{0} \beta \beta$ decay is possible and where there are no contradictions with the LEP data. A $2 \beta$-decay model that involves the emission of two Majorons was proposed within supersymmetric theories [23] and several other models of the Majoron were proposed in the 1990s. By the term "Majoron", one means massless or light bosons that are associated with neutrinos. In these models, the Majoron can carry a lepton charge and is not required to be a Goldstone boson [24]. A decay process that involves the emission of two "Majorons" is also possible [25]. In models featuring a vector Majoron, the Majoron is the longitudinal component of a massive gauge boson emitted in $2 \beta$ decay [26]. For the sake of simplicity, each such object is referred to here as a Majoron. In the Ref. [27], a "bulk" Majoron model was proposed in the context of the "brane-bulk" scenario for particle physics.

The possible two electrons energy spectra for different $2 \beta$ decay modes of ${ }^{100}$ Mo are shown in Fig. 1. Here $\mathrm{n}$ is the spectral index, which defines the shape of the spectrum. For example, for an ordinary Majoron $\mathrm{n}=1$, for $2 \nu$ decay $\mathrm{n}=5$, in the case of a bulk Majoron $\mathrm{n}=2$ and for the process with two Majoron emission $n=3$ or 7 . 


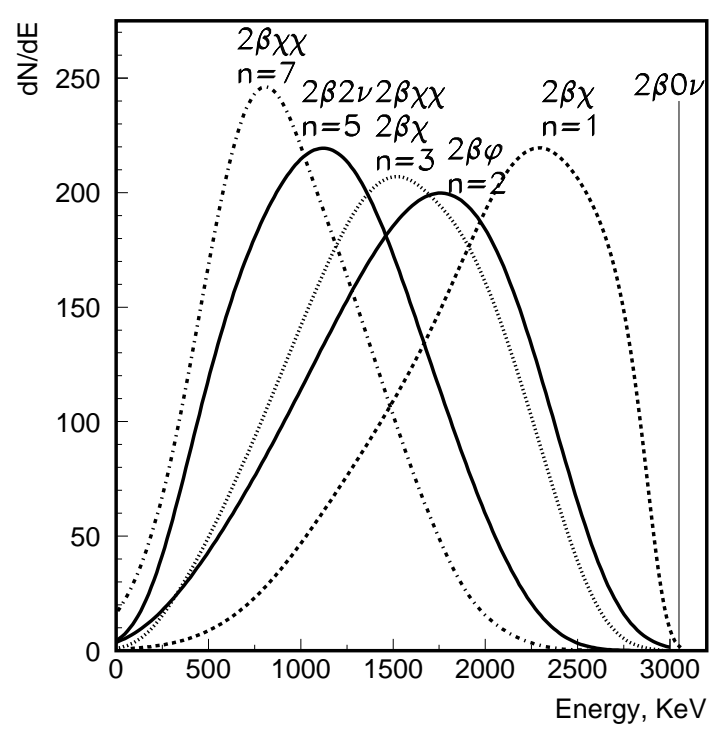

Figure 1. Energy spectra of different modes of $2 \nu \beta \beta(n=5), 0 \nu \chi^{0} \beta \beta(n=1,2$ and 3$)$ and $0 \nu \chi^{0} \chi^{0} \beta \beta(n=3$ and 7$)$ decays of ${ }^{100} \mathrm{Mo}$.

\section{RESULTS OF EXPERIMENTAL INVESTIGATIONS}

The number of possible candidates for double-beta decay is quite large, there are 35 nuclei. ${ }^{2}$ However, nuclei for which the double-beta-transition energy $\left(E_{2 \beta}\right)$ is in excess of 2 $\mathrm{MeV}$ are of greatest interest, since the double-beta-decay probability strongly depends on the transition energy $\left(\sim E_{2 \beta}^{11}\right.$ for $2 \nu \beta \beta$ decay, $\sim E_{2 \beta}^{7}$ for $0 \nu \chi^{0} \beta \beta$ decay and $\sim E_{2 \beta}^{5}$ for $0 \nu \beta \beta$ decay). In transitions to excited states of the daughter nucleus, the excitation energy is removed via the emission of one or more photons, which can be detected, and this can serve as an additional source of information about double-beta decay. As an example Fig. 2 shows the diagram of energy levels in the ${ }^{100} \mathrm{Mo}-{ }^{100} \mathrm{Tc}-{ }^{100} \mathrm{Ru}$ nuclear triplet.

\subsection{Two neutrino double beta decay}

This decay was first recorded in 1950 in a geochemical experiment with ${ }^{130} \mathrm{Te}$ [29]; in 1967, $2 \nu \beta \beta$ decay was found for ${ }^{82} \mathrm{Se}$ also in a geochemical experiment [30]. Attempts to observe this decay in a direct experiment employing counters had been futile for a long time.

\footnotetext{
${ }^{2}$ In addition 34 nuclei can undergo double electron capture, while twenty two nuclei and six nuclei can undergo, respectively, $\mathrm{EC} \beta^{+}$and $2 \beta^{+}$decay (see the tables in [28]).
} 


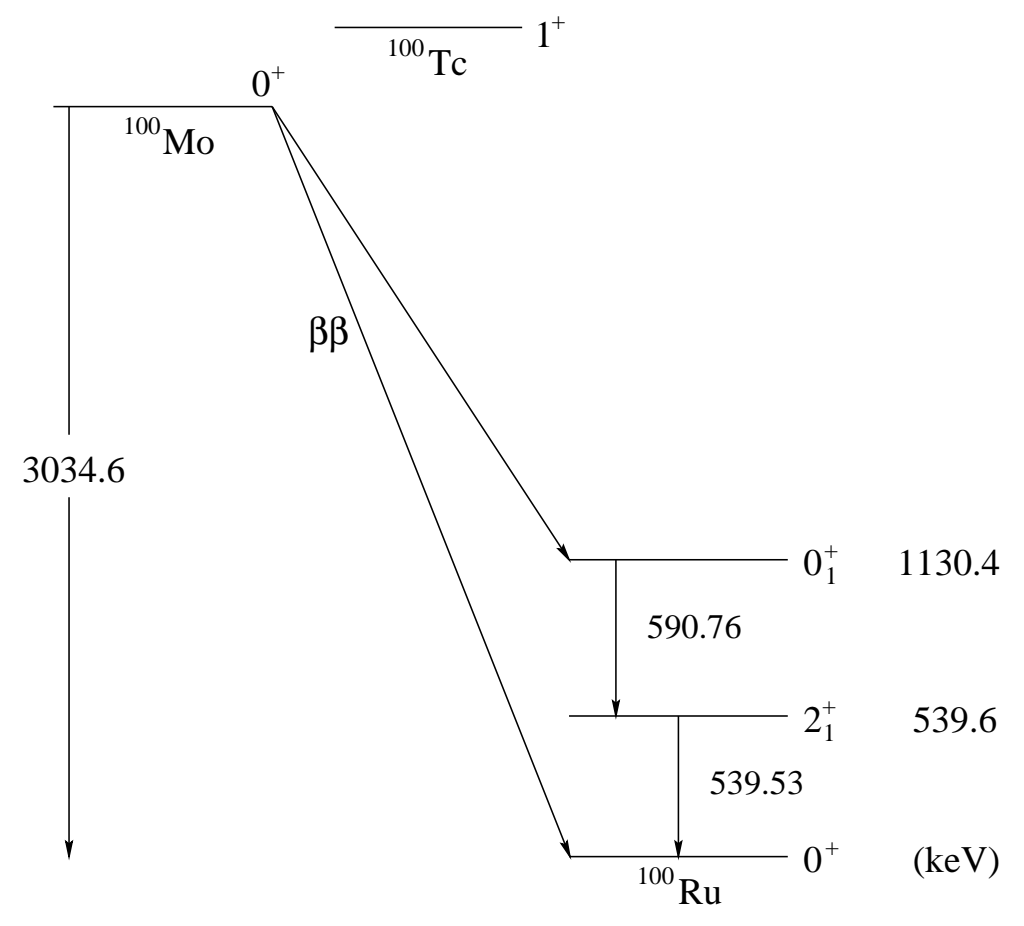

Figure 2. Levels scheme for ${ }^{100} \mathrm{Mo}-{ }^{100} \mathrm{Tc}-{ }^{100} \mathrm{Ru}$.

Only in 1987 could M. Moe, who used a time-projection chamber (TPC), observe $2 \nu \beta \beta$ decay in ${ }^{82} \mathrm{Se}$ for the first time [31]. In the next few years, experiments were able to detect $2 \nu \beta \beta$ decay in many nuclei. In ${ }^{100} \mathrm{Mo}$ and ${ }^{150} \mathrm{Nd} 2 \beta(2 \nu)$ decay to the $0^{+}$excited state of the daughter nucleus was measured too (see section 2.4). Also, the $2 \nu \beta \beta$ decay of ${ }^{238} \mathrm{U}$ was detected in a radiochemical experiment [32], and in a geochemical experiment the ECEC process was detected in ${ }^{130} \mathrm{Ba}$ (see section 2.5). Table 1 displays the present-day averaged and recommended values of $\mathrm{T}_{1 / 2}(2 \nu)$ from [33, 34]. At present, experiments devoted to detecting $2 \nu \beta \beta$ decay are approaching a level where it is insufficient to just record the decay. It is necessary to measure numerous parameters of this process to a high precision (energy sum spectrum, single electron energy spectrum and angular distribution). Tracking detectors that are able to record both the energy of each electron and the angle at which they diverge are the most appropriate instruments for solving this problem. 
Table 1. Average and recommended $T_{1 / 2}(2 \nu)$ values (from $[33,34]$ ).

\begin{tabular}{cc}
\hline Isotope & $T_{1 / 2}(2 \nu), \mathrm{y}$ \\
\hline${ }^{48} \mathrm{Ca}$ & $4.3_{-1.0}^{+2.1} \cdot 10^{19}$ \\
${ }^{76} \mathrm{Ge}$ & $(1.5 \pm 0.1) \cdot 10^{21}$ \\
${ }^{82} \mathrm{Se}$ & $(0.92 \pm 0.07) \cdot 10^{20}$ \\
${ }^{96} \mathrm{Zr}$ & $(2.0 \pm 0.3) \cdot 10^{19}$ \\
${ }^{100} \mathrm{Mo}$ & $(7.1 \pm 0.4) \cdot 10^{18}$ \\
${ }^{100} \mathrm{Mo}{ }^{100} \mathrm{Ru}\left(0_{1}^{+}\right)$ & $\left(6.2_{-0.7}^{+0.9}\right) \cdot 10^{20}$ \\
${ }^{116} \mathrm{Cd}$ & $(3.0 \pm 0.2) \cdot 10^{19}$ \\
${ }^{128} \mathrm{Te}$ & $(2.5 \pm 0.3) \cdot 10^{24}$ \\
${ }^{130} \mathrm{Te}$ & $(0.9 \pm 0.1) \cdot 10^{21}$ \\
${ }^{150} \mathrm{Nd}$ & $(7.8 \pm 0.7) \cdot 10^{18}$ \\
${ }^{150} \mathrm{Nd}-{ }^{150} \mathrm{Sm}\left(0_{1}^{+}\right)$ & $1.4_{-0.4}^{+0.5} \cdot 10^{20}$ \\
${ }^{238} \mathrm{U}$ & $(2.0 \pm 0.6) \cdot 10^{21}$ \\
${ }^{130} \mathrm{Ba} ; \mathrm{ECEC}(2 \nu)$ & $(2.2 \pm 0.5) \cdot 10^{21}$ \\
\hline
\end{tabular}

\subsection{Neutrinoless double beta decay}

In contrast to two-neutrino decay, neutrinoless double-beta decay has not yet been observed ${ }^{3}$, although it is easier to detect it. In this case, one seeks, in the experimental spectrum, a peak of energy equal to the double-beta- transition energy and of width determined by the detector's resolution.

The constraints on the existence of $0 \nu \beta \beta$ decay are presented in Table 2 for the nuclei that are the most promising candidates. In calculating constraints on $\left\langle m_{\nu}\right\rangle$, the nuclear matrix elements from [10-13] were used (3-d column). It is advisable to employ the calculations from these studies, because the calculations are the most thorough and take into account

\footnotetext{
${ }^{3}$ The possible exception is the result with ${ }^{76} \mathrm{Ge}$, published by a fraction of the Heidelberg-Moscow Collaboration, $T_{1 / 2} \simeq 1.2 \cdot 10^{25}$ y [35] or $T_{1 / 2} \simeq 2.2 \cdot 10^{25}$ y [36] (see Table 2). First time the "positive" result was mentioned in [37]. The Moscow part of the Collaboration does not agree with this conclusion [38] and there are others who are critical of this result [39-41]. Thus, at the present time, this "positive" result is not accepted by the " $2 \beta$ decay community" and it has to be checked by new experiments.
} 
Table 2. Best present results on $0 \nu \beta \beta$ decay (limits at $90 \%$ C.L.). ${ }^{*}$ ) See footnote ${ }^{3}$; ${ }^{* *}$ current experiments; ${ }^{* * *}$ conservative limit from [48] is presented.

\begin{tabular}{lcccc}
\hline Isotope & $T_{1 / 2}, \mathrm{y}$ & $\left\langle m_{\nu}\right\rangle, \mathrm{eV}$ & $\left\langle m_{\nu}\right\rangle, \mathrm{eV}$ & Experiment \\
$\left(E_{2 \beta}, \mathrm{keV}\right)$ & & {$[10-13]$} & {$[42]$} & \\
\hline${ }^{76} \mathrm{Ge}(2039)$ & $>1.9 \cdot 10^{25}$ & $<0.22-0.41$ & $<0.69$ & HM [43] \\
& $\simeq 1.2 \cdot 10^{25}(?)^{*)}$ & $\simeq 0.28-0.52(?)^{*)}$ & $\simeq 0.87(?)^{*)}$ & Part of HM [35] \\
& $\simeq 2.2 \cdot 10^{25}(?)^{*)}$ & $\simeq 0.21-0.38(?)^{*)}$ & $\simeq 0.64(?)^{*)}$ & Part of HM [36] \\
& $>1.6 \cdot 10^{25}$ & $<0.24-0.44$ & $<0.75$ & IGEX [44] \\
\hline${ }^{130} \mathrm{Te}(2529)$ & $>3 \cdot 10^{24}$ & $<0.29-0.57$ & $<0.75$ & CUORICINO ${ }^{* *)}[46]$ \\
${ }^{100} \mathrm{Mo}(3034)$ & $>5.8 \cdot 10^{23}$ & $<0.61-1.28$ & - & NEMO- $\left.3^{* *}\right)$ \\
${ }^{136} \mathrm{Xe}(2458)$ & $>4.5 \cdot 10^{23 * * *)}$ & $<1.14-2.68$ & $<2.2$ & DAMA [48] \\
${ }^{82} \mathrm{Se}(2995)$ & $>2.1 \cdot 10^{23}$ & $<1.16-2.17$ & $<3.4$ & NEMO-3 $\left.{ }^{* * *}\right)$ \\
${ }^{116} \mathrm{Cd}(2805)$ & $>1.7 \cdot 10^{23}$ & $<1.40-2.76$ & $<1.8$ & SOLOTVINO [49] \\
\hline
\end{tabular}

the most recent theoretical achievements. In these papers $g_{p p}$ values $\left(g_{p p}\right.$ is parameter of the QRPA theory) were fixed using experimental half-life values for $2 \nu$ decay and then $\operatorname{NME}(0 \nu)$ were calculated. In column four, limits on $\left\langle m_{\nu}\right\rangle$, which were obtained using the NMEs from a recent Shell Model (SM) calculations [42].

From Table 2 using NME values from [10-13], the limits on $\left\langle m_{\nu}\right\rangle$ for ${ }^{130}$ Te are comparable with the ${ }^{76} \mathrm{Ge}$ results. Now one cannot select any experiment as the best one. The assemblage of sensitive experiments for different nuclei permits one to increase the reliability of the limit on $\left\langle m_{\nu}\right\rangle$. Present conservative limit can be set as $0.75 \mathrm{eV}$.

\subsection{Double beta decay with Majoron emission}

Table 3 displays the best present-day constraints for an "ordinary" Majoron $(\mathrm{n}=1)$. The "nonstandard" models of the Majoron were experimentally tested in [52] for ${ }^{76}$ Ge and in [53] for ${ }^{100} \mathrm{Mo},{ }^{116} \mathrm{Cd},{ }^{82} \mathrm{Se}$, and ${ }^{96} \mathrm{Zr}$. Constraints on the decay modes involving the emission of two Majorons were also obtained for ${ }^{100} \mathrm{Mo}$ [54], ${ }^{116} \mathrm{Cd}$ [49], and ${ }^{130} \mathrm{Te}$ [55]. In 
Table 3. Best present limits on $0 \nu \chi^{0} \beta \beta$ decay (ordinary Majoron) at $90 \%$ C.L. The NME from the following works were used, 3-d column: [10-13], 4-th column: [42]. *) Conservative limit from [48] is presented.

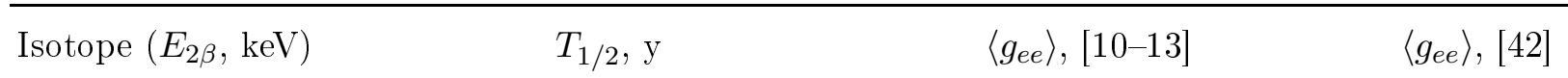

\begin{tabular}{lccc}
\hline${ }^{76} \mathrm{Ge}(2039)$ & $>6.4 \cdot 10^{22}[43]$ & $<(0.54-1.44) \cdot 10^{-4}$ & $<2.4 \cdot 10^{-4}$ \\
${ }^{82} \mathrm{Se}(2995)$ & $>1.5 \cdot 10^{22}[50]$ & $<(0.58-1.19) \cdot 10^{-4}$ & $<1.9 \cdot 10^{-4}$ \\
${ }^{100} \mathrm{Mo}(3034)$ & $>2.7 \cdot 10^{22}[50]$ & $<(0.35-0.85) \cdot 10^{-4}$ & - \\
${ }^{116} \mathrm{Cd}(2805)$ & $>8 \cdot 10^{21}[49]$ & $<(0.79-2.56) \cdot 10^{-4}$ & $<1.7 \cdot 10^{-4}$ \\
${ }^{128} \mathrm{Te}(867)$ & $>2 \cdot 10^{24}($ geochem $)[51]$ & $<(0.61-0.97) \cdot 10^{-4}$ & $<1.4 \cdot 10^{-4}$ \\
${ }^{136} \mathrm{Xe}(2458)$ & $>1.6 \cdot 10^{22 *)}[48]$ & $<(1.51-3.54) \cdot 10^{-4}$ & $<2.9 \cdot 10^{-4}$ \\
\hline
\end{tabular}

a recent NEMO Collaboration paper [50], new results for these processes in ${ }^{100} \mathrm{Mo}$ and ${ }^{82} \mathrm{Se}$ were obtained with the NEMO-3 detector. Table 4 gives the best experimental constraints on decays accompanied by the emission of one or two Majorons (for $\mathrm{n}=2$, 3, and 7). Hence at the present time only limits on double beta decay with Majoron emission have been obtained (see table 3 and 4). A conservative present limit on the coupling constant of ordinary Majoron to the neutrino is $\left\langle g_{e e}\right\rangle<1.9 \cdot 10^{-4}$.

\subsection{Double beta decay to the excited states}

The $\beta \beta$ decay can proceed through transitions to the ground state as well as to various excited states of the daughter nucleus. Studies of the latter transitions allow supplementary information about $\beta \beta$ decay. The first experimental studies of $\beta \beta$ decay to the excited state were done by E. Fiorini in 1977 [56]. It was an aside to his experiment with ${ }^{76} \mathrm{Ge}$ (transition to $0^{+}$ground state). The first experimental work designed to investigate $\beta \beta$ decay to the excited states was done in 1982 [57]. In 1989 it was proposed that using low-background facilities utilizing High Purity Germanium (HPGe) detectors, the $2 \nu \beta \beta$ decay to the $0_{1}^{+}$level in the daughter nucleus may be detected for such nuclei as ${ }^{100} \mathrm{Mo},{ }^{96} \mathrm{Zr}$ and ${ }^{150} \mathrm{Nd}$ [58]. Soon 
Table 4. Best present limits on $T_{1 / 2}$ for decay with one and two Majorons at $90 \%$ C.L. for modes with spectral index $\mathrm{n}=2, \mathrm{n}=3$ and $\mathrm{n}=7$.

\begin{tabular}{lccc}
\hline Isotope $\left(E_{2 \beta}, \mathrm{keV}\right)$ & $\mathrm{n}=2$ & $\mathrm{n}=3$ & $\mathrm{n}=7$ \\
\hline${ }^{76} \mathrm{Ge}(2039)$ & - & $>5.8 \cdot 10^{21}[52]$ & $>6.6 \cdot 10^{21}[52]$ \\
${ }^{82} \mathrm{Se}(2995)$ & $>6 \cdot 10^{21}[50]$ & $>3.1 \cdot 10^{21}[50]$ & $>5 \cdot 10^{20}[50]$ \\
${ }^{96} \mathrm{Zr}(3550)$ & - & $>6.3 \cdot 10^{19}[53]$ & $>2.4 \cdot 10^{19}[53]$ \\
${ }^{100} \mathrm{Mo}(3034)$ & $>1.7 \cdot 10^{22}[50]$ & $>1 \cdot 10^{22}[50]$ & $>7 \cdot 10^{19}[50]$ \\
${ }^{116} \mathrm{Cd}(2805)$ & $>1.7 \cdot 10^{21}[49]$ & $>8 \cdot 10^{20}[49]$ & $>3.1 \cdot 10^{19}[49]$ \\
${ }^{130} \mathrm{Te}(2529)$ & - & $>9 \cdot 10^{20}[55]$ & - \\
${ }^{128} \mathrm{Te}(867)(\mathrm{geochem})$ & $>2 \cdot 10^{24}[51]$ & $>2 \cdot 10^{24}[51]$ & $>2 \cdot 10^{24}[51]$ \\
\hline
\end{tabular}

after this double beta decay of ${ }^{100}$ Mo to the $0^{+}$excited state at $1130.29 \mathrm{keV}$ in ${ }^{100} \mathrm{Ru}$ was observed [59]. This result was confirmed in independent experiments with HPGe detectors [60-62]. Recently the $2 \nu \beta \beta$ decay of ${ }^{100} \mathrm{Mo}$ to the $0_{1}^{+}$level in ${ }^{100} \mathrm{Ru}$ was detected with the tracking detector NEMO-3 where all the decay products (two electrons and two $\gamma$-rays) were detected and hence all the information about the decay was obtained (total energy spectrum, single electron spectrum, single $\gamma$ spectrum and angular distributions) [63]. In 2004 this transition was detected in ${ }^{150} \mathrm{Nd}$ [64]. During the last 15 years new limits for many nuclei and different modes of decay to the excited states were established (see reviews $[65,66])$. Present motivations to do this search are the following:

1) Nuclear spectroscopy (to know decay schemes of nuclei)

2) Nuclear matrix elements

3) Examination of some new ideas (such as the "bosonic" component of the neutrino $[17,18])$

4) Neutrino mass investigations:

a) $0 \nu \beta \beta\left(0^{+}-0_{1}^{+}\right)$decay; in this case one has a very nice signature for the decay and hence high sensitivity to neutrino mass can be reached

b) High sensitivity to the effective Majorana neutrino mass can be reached in the case of the ECEC $(0 \nu)$ transition if the resonance condition is realized (see section 2.5.1). 
Table 5. Best present limits on $2 \nu \beta \beta$ transition to the $2_{1}^{+}$excited state (90\% C.L.).

\begin{tabular}{lcccc}
\hline Isotope & $\mathrm{E}_{2 \beta}, \mathrm{keV}$ & Experiment $T_{1 / 2}, \mathrm{y}$ & Theory [67] & Theory [68, 69] \\
\hline${ }^{48} \mathrm{Ca}$ & 3288.5 & $>1.8 \times 10^{20}[70]$ & $1.7 \times 10^{24}$ & - \\
${ }^{150} \mathrm{Nd}$ & 3033.6 & $>9.1 \times 10^{19}[71]$ & - & - \\
${ }^{96} \mathrm{Zr}$ & 2572.2 & $>7.9 \times 10^{19}[72]$ & $2.3 \times 10^{25}$ & $(3.8-4.8) \times 10^{21}$ \\
${ }^{100} \mathrm{Mo}$ & 2494.5 & $>1.6 \times 10^{21}[59]$ & $1.2 \times 10^{25}$ & $3.4 \times 10^{22}[73]$ \\
${ }^{82} \mathrm{Se}$ & 2218.5 & $>1.4 \times 10^{21}[74]$ & - & $2.8 \times 10^{23}-3.3 \times 10^{26}$ \\
${ }^{130} \mathrm{Te}$ & 1992.7 & $>2.8 \times 10^{21}[75]$ & $6.9 \times 10^{26}$ & $(3.0-27) \times 10^{22}$ \\
${ }^{116} \mathrm{Cd}$ & 1511.5 & $>2.3 \times 10^{21}[76]$ & $3.4 \times 10^{26}$ & $1.1 \times 10^{24}$ \\
${ }^{76} \mathrm{Ge}$ & 1480 & $>1.1 \times 10^{21}[77]$ & $5.8 \times 10^{28}$ & $(7.8-10) \times 10^{25}$ \\
\hline
\end{tabular}

$2 \nu \beta \beta$ transition to $2_{1}^{+}$excited state. The $2 \nu \beta \beta$ decay to the $2_{1}^{+}$excited state is strongly suppressed and practically inaccessible to detection. However, for a few nuclei $\left({ }^{96} \mathrm{Zr},{ }^{100} \mathrm{Mo}\right.$, $\left.{ }^{130} \mathrm{Te}\right)$ there are some "optimistic" predictions for half-lives $\left(T_{1 / 2} \sim 10^{22}-10^{24} \mathrm{y}\right)$ and there is a chance to detect such decays in the next generation of the double beta decay experiments. The best present limits are given in Table 5 .

$2 \nu \beta \beta$ transition to $0_{1}^{+}$excited state. This transition has been detected in ${ }^{100} \mathrm{Mo}$ and ${ }^{150} \mathrm{Nd}$. The best results and limits are presented in Table 6. One can conclude that there is a good chance to detect this type of decay in ${ }^{96} \mathrm{Zr},{ }^{82} \mathrm{Se},{ }^{116} \mathrm{Cd},{ }^{130} \mathrm{Te}$ and ${ }^{76} \mathrm{Ge}$. Table 7 presents all the existing positive results for $2 \nu \beta \beta$ decay of ${ }^{100}$ Mo to the first $0^{+}$excited state of ${ }^{100} \mathrm{Ru}$. The half-life averaged over four experiments is given in the bottom row, $T_{1 / 2}=6.2_{-0.7}^{+0.9} \times 10^{20} \mathrm{y}$.

$0 \nu \beta \beta$ transition to $2_{1}^{+}$excited state. The $0 \nu \beta \beta\left(0^{+}-2_{1}^{+}\right)$decay had long been accepted to be possible because of the contribution of right-handed currents and is not sensitive to the neutrino mass contribution. However, it was demonstrated [81] that the relative sensitivities of $\left(0^{+}-2_{1}^{+}\right)$decays to the neutrino mass $\left\langle m_{\nu}\right\rangle$ and the right-handed current $\langle\eta\rangle$ are comparable to those of $0 \nu \beta \beta$ decay to the ground state. At the same time, the $\left(0^{+}-2_{1}^{+}\right)$ decay is more sensitive to $\langle\lambda\rangle$. The best present experimental limits are giwen in Table 8 .

$0 \nu \beta \beta$ transition to $0_{1}^{+}$excited state. The $0 \nu \beta \beta$ transition to the $0^{+}$excited states of the 
Table 6. Best present results and limits on $2 \nu \beta \beta$ transition to the $0_{1}^{+}$excited state. Limits are given at the $90 \%$ C.L. $\left.{ }^{*}\right)$ Corrected value is used (see remark in [80]).

\begin{tabular}{lcccc}
\hline Isotope & $\mathrm{E}_{2 \beta}, \mathrm{keV}$ & Experiment $T_{1 / 2}, \mathrm{y}$ & Theory $[68,69]$ & Theory [73] \\
\hline${ }^{150} \mathrm{Nd}$ & 2627.1 & $=1.4_{-0.4}^{+0.5} \times 10^{20}[64]$ & - & - \\
${ }^{96} \mathrm{Zr}$ & 2202.5 & $>6.8 \times 10^{19}[72]$ & $(2.4-2.7) \times 10^{21}$ & $3.8 \times 10^{21}$ \\
${ }^{100} \mathrm{Mo}$ & 1903.7 & $=6.2_{-0.7}^{+0.9} \times 10^{20}$ & $1.6 \times 10^{21}[78]$ & $2.1 \times 10^{21}$ \\
${ }^{82} \mathrm{Se}$ & 1507.5 & $>3.0 \times 10^{21}[74]$ & $(1.5-3.3) \times 10^{21}$ & - \\
${ }^{48} \mathrm{Ca}$ & 1274.8 & $>1.5 \times 10^{20}[70]$ & - & - \\
${ }^{116} \mathrm{Cd}$ & 1048.2 & $>2.0 \times 10^{21}[76]$ & $1.1 \times 10^{22}$ & $1.1 \times 10^{21}$ \\
${ }^{76} \mathrm{Ge}$ & 916.7 & $>6.2 \times 10^{21}[79]$ & $(7.5-310) \times 10^{21}$ & $4.5 \times 10^{21}$ \\
${ }^{130} \mathrm{Te}$ & 735.3 & $>2.3 \times 10^{21}[80]$ & $(5.1-14) \times 10^{22 *)}$ & - \\
\hline
\end{tabular}

Table 7. Present "positive" results on $2 \nu \beta \beta$ decay of ${ }^{100}$ Mo to the first $0^{+}$excited state of ${ }^{100} \mathrm{Ru}(1130.4$ $\mathrm{keV}) . \mathrm{N}$ is the number of useful events, $\mathrm{S} / \mathrm{B}$ is the signal-to-background ratio.

\begin{tabular}{lcccc}
\hline$T_{1 / 2}, \mathrm{y}$ & $\mathrm{N}$ & $\mathrm{S} / \mathrm{B}$ & Year, references & Wethod \\
\hline $6.1_{-1.1}^{+1.8} \times 10^{20}$ & 133 & $\sim 1 / 7$ & $1995[59]$ & HPGe \\
$9.3_{-1.7}^{+2.8} \pm 1.4 \times 10^{20}$ & 154 & $\sim 1 / 4$ & $1999[60]$ & HPGe \\
$6.0_{-1.1}^{+1.9} \pm 0.6 \times 10^{20}$ & 19.5 & $8 / 1$ & $2001[61,62]$ & $2 \times H P G e$ \\
$5.7_{-0.9}^{+1.3} \pm 0.8 \times 10^{20}$ & 37.5 & $3 / 1$ & $2007[63]$ & NEMO-3 \\
\hline
\end{tabular}

Average value: $6.2_{-0.7}^{+0.9} \times 10^{20} \mathrm{y}$

daughter nuclei provides a clear-cut signature. In addition to two electrons with a fixed total energy, there are two photons, whose energies are strictly fixed as well. In a hypothetical experiment detecting all decay products with high efficiency and high energy resolution, the background can be reduced to nearly zero. It is possible this idea will be used in future experiments featuring a large mass of the isotope under study (as mentioned in Refs. [65, 66, 85]). In Ref. [86] it was mentioned that detection of this transition will give us the additional possibility to distinguish the $0 \nu \beta \beta$ mechanisms. The best present limits are 
Table 8. Best present limits on $0 \nu \beta \beta$ transition to the $2_{1}^{+}$excited state (90\% C.L.).

\begin{tabular}{lcccc}
\hline Isotope & $\mathrm{E}_{2 \beta}, \mathrm{keV}$ & Experiment $T_{1 / 2}, \mathrm{y}$ & Theory $[81],\left\langle m_{\nu}\right\rangle=1 \mathrm{eV}$ & Theory [81], $\langle\lambda\rangle=10^{-6}$ \\
\hline${ }^{76} \mathrm{Ge}$ & 1480 & $>8.2 \times 10^{23}[82]$ & $8.2 \times 10^{31}$ & $6.5 \times 10^{29}$ \\
${ }^{100} \mathrm{Mo}$ & 2494.5 & $>1.6 \times 10^{23}[63]$ & $6.8 \times 10^{30}$ & $2.1 \times 10^{27}$ \\
${ }^{130} \mathrm{Te}$ & 1992.7 & $>1.4 \times 10^{23}[55]$ & - & - \\
${ }^{116} \mathrm{Cd}$ & 1511.5 & $>2.9 \times 10^{22}[49]$ & - & - \\
${ }^{136} \mathrm{Xe}$ & 1649.4 & $>6.5 \times 10^{21}[83]$ & - & - \\
${ }^{82} \mathrm{Se}$ & 2218.5 & $>2.8 \times 10^{21}[84]$ & - & \\
\hline
\end{tabular}

Table 9. Best present limits on $0 \nu \beta \beta$ transition to the $0_{1}^{+}$excited state (90\% C.L.). Theoretical predictions are given for $\left\langle m_{\nu}\right\rangle=1 \mathrm{eV}$.

\begin{tabular}{lcccc}
\hline Isotope & $\mathrm{E}_{2 \beta}, \mathrm{keV}$ & Experiment, $T_{1 / 2}, \mathrm{y}$ & Theory $[85,88-90]$ & Theory [91] \\
\hline${ }^{150} \mathrm{Nd}$ & 2627.1 & $>1.0 \times 10^{20}[71]$ & - & - \\
${ }^{96} \mathrm{Zr}$ & 2202.5 & $>6.8 \times 10^{19}[72]$ & $2.4 \times 10^{24}$ & - \\
${ }^{100} \mathrm{Mo}$ & 1903.7 & $>8.9 \times 10^{22}[63]$ & $2.6 \times 10^{26}$ & $1.5 \times 10^{25}$ \\
${ }^{82} \mathrm{Se}$ & 1507.5 & $>3.0 \times 10^{21}[74]$ & $9.5 \times 10^{26}$ & $4.5 \times 10^{25}$ \\
${ }^{48} \mathrm{Ca}$ & 1274.8 & $>1.5 \times 10^{20}[70]$ & - & - \\
${ }^{116} \mathrm{Cd}$ & 1048.2 & $>1.4 \times 10^{22}[49]$ & $1.5 \times 10^{27}$ & - \\
${ }^{76} \mathrm{Ge}$ & 916.7 & $>1.3 \times 10^{22}[87]$ & $4.9 \times 10^{26}$ & $2.4 \times 10^{26}$ \\
${ }^{130} \mathrm{Te}$ & 735.3 & $>3.1 \times 10^{22}[55]$ & $7.5 \times 10^{25}$ & - \\
\hline
\end{tabular}

presented in Table 9.

\section{5. $2 \beta^{+}, E C \beta^{+}$, and ECEC processes}

Much less attention has been given to the investigation of $2 \beta^{+}, \beta^{+} \mathrm{EC}$ and ECEC processes although such attempts were done from time to time in the past (see review [66]). Again, the main interest here is connected with neutrinoless decay: 


$$
\begin{gathered}
(A, Z) \rightarrow(A, Z-2)+2 e^{+} \\
e^{-}+(A, Z) \rightarrow(A, Z-2)+e^{+}+X \\
e^{-}+e^{-}+(A, Z) \rightarrow(A, Z-2)^{*} \rightarrow(A, Z-2)+\gamma+2 X
\end{gathered}
$$

There are 34 candidates for these processes. Only 6 nuclei can undergo all the above mentioned processes and 16 nuclei can undergo $\beta^{+}$EC and ECEC while 12 can undergo only ECEC. Detection of the neutrinoless mode in the above processes enable one to determine the effective Majorana neutrino mass $\left\langle m_{\nu}\right\rangle$, parameters of right-handed current admixture in electroweak interaction $(\langle\lambda\rangle$ and $\langle\eta\rangle)$, etc.

Process (4) has a very nice signature because, in addition to two positrons, four annihilation $511 \mathrm{keV}$ gamma quanta will be detected. On the other hand, the rate for this process should be much lower in comparison with $0 \nu \beta \beta$ decay because of substantially lower kinetic energy available in such a transition $(2.044 \mathrm{MeV}$ is spent for creation of two positrons) and of the Coulomb barrier for positrons. There are only six candidates for this type of decay: ${ }^{78} \mathrm{Kr},{ }^{96} \mathrm{Ru},{ }^{106} \mathrm{Cd},{ }^{124} \mathrm{Xe},{ }^{130} \mathrm{Ba}$ and ${ }^{136} \mathrm{Ce}$. The half-lives of most prospective isotopes are estimated to be $\sim 10^{27}-10^{28}$ y (for $\left.\left\langle m_{\nu}\right\rangle=1 \mathrm{eV}\right)[90,92]$; this is approximately $10^{3}-10^{4}$ times higher than for $0 \nu \beta \beta$ decay for such nuclei as ${ }^{76} \mathrm{Ge},{ }^{100} \mathrm{Mo},{ }^{82} \mathrm{Se}$ and ${ }^{130} \mathrm{Te}$.

Process (5) has a nice signature (positron and two annihilation $511 \mathrm{keV}$ gammas) and is not as strongly suppressed as $2 \beta^{+}$decay. In this case, half-life estimates for the best nuclei give $\sim 10^{26}-10^{27} \mathrm{y}$ (again for $\left\langle m_{\nu}\right\rangle=1 \mathrm{eV}$ ) [90, 92].

In the last case (process (6)), the atom de-excites emitting two X-rays and the nucleus de-excites emitting one $\gamma$-ray (bremsstrahlung photon) ${ }^{4}$ For a transition to an excited state of the daughter nucleus, besides a bremsstrahlung photon, $\gamma$-rays are emitted from the decay of the excited state. Thus, there is a clear signature for this process. The rate is practically independent of decay energy and increases with both decreasing bremsstrahlung

\footnotetext{
${ }^{4}$ In fact the processes with irradiation of inner conversion electron, $e^{+} e^{-}$pair or two gammas are also possible [93] (in addition, see discussion in [94]). These possibilities are especially important in the case of the $\operatorname{ECEC}(0 \nu)$ transition with the capture of two electrons from the $\mathrm{K}$ shell. In this case the transition with irradiation of one $\gamma$ is strongly suppressed [93].
} 
photon energy and increasing Z [94, 95]. The rate is quite low even for heavy nuclei, with $T_{1 / 2} \sim 10^{28}-10^{31} \mathrm{y}\left(\left\langle m_{\nu}\right\rangle=1 \mathrm{eV}\right)[94]$. The rate can be increased in $\sim 10^{6}$ times if resonance conditiones exist (see Section 2.5.1).

For completeness, let us present the two-neutrino modes of $2 \beta^{+}, \beta^{+} \mathrm{EC}$ and ECEC processes:

$$
\begin{gathered}
(A, Z) \rightarrow(A, Z-2)+2 e^{+}+2 \nu \\
e^{-}+(A, Z) \rightarrow(A, Z-2)+e^{+}+2 \nu+X \\
e^{-}+e^{-}+(A, Z) \rightarrow(A, Z-2)+2 \nu+2 X
\end{gathered}
$$

These processes are not forbidden by any conservation laws, and their observation is interesting from the point of view of investigating nuclear-physics aspects of double-beta decay. Processes (7) and (8) are quite strongly suppressed because of low phase-space volume, and investigation of process (9) is very difficult because one only has low energy X-rays to detect. In the case of double-electron capture, it is again interesting to search for transitions to the excited states of daughter nuclei, which are easier to detect experimentally [96]. For the best candidates half-life is estimated as $\sim 10^{27} \mathrm{y}$ for $\beta^{+} \beta^{+}, \sim 10^{22} \mathrm{y}$ for $\beta^{+} \mathrm{EC}$ and $\sim 10^{21} \mathrm{y}$ for ECEC process [92].

During the last few years, interest in the $\beta^{+} \beta^{+}, \beta^{+} \mathrm{EC}$ and ECEC processes has greatly increased. For the first time a positive result was obtained in a geochemical experiment with ${ }^{130} \mathrm{Ba}$, where the $\operatorname{ECEC}(2 \nu)$ process was detected with a half-life of $(2.2 \pm 0.5) \times 10^{21}$ y [97]. Recently new limits on the $\operatorname{ECEC}(2 \nu)$ process in the promising candidate isotopes $\left({ }^{78} \mathrm{Kr}\right.$ and $\left.{ }^{106} \mathrm{Cd}\right)$ were established $\left(1.5 \times 10^{21}\right.$ y [98] and $2 \times 10^{20}$ y [99], respectively). Very recently $\beta^{+} \mathrm{EC}$ and ECEC processes in ${ }^{120} \mathrm{Te}[100,101],{ }^{74} \mathrm{Se}[102],{ }^{64} \mathrm{Zn}[103,104]$ and ${ }^{112} \mathrm{Sn}$ [104-106] were investigated. Among the recent papers there are a few new theoretical papers with half-life estimations [90, 108-111]. Nevertheless the $\beta^{+} \beta^{+}, \beta^{+} \mathrm{EC}$ and ECEC processes have not been investigated very well theoretically or experimentally. One can imagine some unexpected results here, which is why any improvements in experimental sensitivity for such transitions has merit.

Table 10 gives a compendium of the best present-day constrains for $2 \beta^{+}, \mathrm{EC} \beta^{+}$, and 
ECEC processes and the result of the geochemical experiment that employed ${ }^{130} \mathrm{Ba}$ and which yields the first indication of the observation of $\operatorname{ECEC}(2 \nu)$ capture.

\subsection{1. $E C E C(O \nu)$ resonance transition to the excited states}

In Ref. [113] it was the first mentioned that in the case of $\operatorname{ECEC}(0 \nu)$ transition a resonance condition could exist for transitions to a "right energy" excited state of the daughter nucleus, when the decay energy is closed to zero. In 1982 the same idea was proposed for transitions to the ground state [114]. In 1983 this transition was discussed for ${ }^{112} \mathrm{Sn}^{-112}{ }^{112}\left(0^{+} ; 1871\right.$ $\mathrm{keV}$ ) [107]. In 2004 the idea was reanalyzed in Ref. [94] and new resonance condition for the decay was formulated. The possible enhancement of the transition rate was estimated as $\sim 10^{6}[94,107]$, which means that the process starts to be competitive with $0 \nu \beta \beta$ decay sensitivity to neutrino mass and it is possible to check this by experiment. There are several candidates for such resonance transitions, to the ground $\left({ }^{152} \mathrm{Gd},{ }^{164} \mathrm{Eu}\right.$ and $\left.{ }^{180} \mathrm{~W}\right)$ and to the excited states $\left({ }^{74} \mathrm{Se},{ }^{78} \mathrm{Kr},{ }^{96} \mathrm{Ru},{ }^{106} \mathrm{Cd},{ }^{112} \mathrm{Sn},{ }^{130} \mathrm{Ba},{ }^{136} \mathrm{Ce}\right.$ and $\left.{ }^{162} \mathrm{Er}\right)$ of daughter nuclei. The precision needed to realize resonance conditions is well below $1 \mathrm{keV}$. To select the best candidate from the above list one will have to know the atomic mass difference with an accuracy better than $1 \mathrm{keV}$. Such measurements are planed for the future. Recently the first experiment to search specially for such a resonance transition in ${ }^{74} \mathrm{Se}_{-}{ }^{74} \mathrm{Ge}\left(2^{+} ; 1204.2\right.$ $\mathrm{keV})$ was performed yielding a limit $T_{1 / 2}>5.5 \times 10^{18}$ y [115]. For the ${ }^{112} \mathrm{Sn}-{ }^{112} \mathrm{Cd}\left(0^{+} ; 1871\right.$ $\mathrm{keV}$ ) transition limit $T_{1 / 2}>0.92 \times 10^{20} \mathrm{y}$ was obtained [105]. It has also been demonstrated

that using enriched ${ }^{112} \mathrm{Sn}$ (or ${ }^{74} \mathrm{Se}$ ) at an installation such as GERDA or MAJORANA a sensitivity on the level $\sim 10^{26}$ y can be reached. The best present limits are presented in Table 11.

\section{BEST CURRENT PRESENT EXPERIMENTS (NEMO-3 AND CUORICINO)}

\subsection{NEMO-3 experiment [47, 120, 121]}

This tracking experiment, in contrast to experiments with ${ }^{76} \mathrm{Ge}$, detects not only the total energy deposition, but other parameters of the process, including the energy of the individual electrons, angle between them, and the coordinates of the event in the source plane. The performance of the detector was studied with the NEMO-2 prototype [123]. Since June of 
Table 10. Most significant experimental results for $2 \beta^{+}, \mathrm{EC} \beta^{+}$, and ECEC processes (all limits are presented at a $90 \%$ C.L.). Here $\mathrm{Q}$ is equal to $\Delta M$ (atomic mass difference of parent and daughter nuclei) for ECEC, $\Delta M-1022 \mathrm{keV}$ for $\mathrm{EC} \beta^{+}$and $\Delta M-2044 \mathrm{keV}$ for $2 \beta^{+}$.

\begin{tabular}{|c|c|c|c|c|}
\hline Decay type & Nucleus & $\mathrm{Q}, \mathrm{keV}$ & $T_{1 / 2}, \mathrm{y}$ & References \\
\hline \multirow[t]{4}{*}{$\operatorname{ECEC}(0 \nu)$} & ${ }^{130} \mathrm{Ba}$ & 2611 & $>4 \times 10^{21}$ & {$[112]$} \\
\hline & ${ }^{78} \mathrm{Kr}$ & 2866 & $>1.5 \times 10^{21}$ & {$[98]$} \\
\hline & ${ }^{132} \mathrm{Ba}$ & 839.9 & $>3 \times 10^{20}$ & {$[112]$} \\
\hline & ${ }^{106} \mathrm{Cd}$ & 2771 & $>0.9 \times 10^{19}$ & {$[49]$} \\
\hline \multirow[t]{6}{*}{$\operatorname{ECEC}(2 \nu)$} & ${ }^{130} \mathrm{Ba}$ & 2611 & $>4 \times 10^{21}$ & [112] \\
\hline & & & $=2.1_{-0.8}^{+3.0} \times 10^{21}$ & {$[112]$} \\
\hline & & & $=(2.2 \pm 0.5) \times 10^{21}$ & {$[97]$} \\
\hline & ${ }^{78} \mathrm{Kr}$ & 2866 & $>1.5 \times 10^{21}$ & {$[98]$} \\
\hline & ${ }^{106} \mathrm{Cd}$ & 2771 & $>2 \times 10^{20}$ & {$[99]$} \\
\hline & ${ }^{132} \mathrm{Ba}$ & 839.9 & $>3 \times 10^{20}$ & {$[112]$} \\
\hline \multirow[t]{5}{*}{$\mathrm{EC} \beta^{+}(0 \nu)$} & ${ }^{130} \mathrm{Ba}$ & 1589 & $>4 \times 10^{21}$ & {$[112]$} \\
\hline & ${ }^{78} \mathrm{Kr}$ & 1844 & $>2.5 \times 10^{21}$ & {$[116]$} \\
\hline & ${ }^{58} \mathrm{Ni}$ & 903.8 & $>4.4 \times 10^{20}$ & {$[118]$} \\
\hline & ${ }^{106} \mathrm{Cd}$ & 1749 & $>3.7 \times 10^{20}$ & {$[117]$} \\
\hline & ${ }^{92} \mathrm{Mo}$ & 627.1 & $>1.9 \times 10^{20}$ & {$[119]$} \\
\hline \multirow[t]{5}{*}{$\mathrm{EC} \beta^{+}(2 \nu)$} & ${ }^{130} \mathrm{Ba}$ & 1589 & $>4 \times 10^{21}$ & {$[112]$} \\
\hline & ${ }^{58} \mathrm{Ni}$ & 903.8 & $>4.4 \times 10^{20}$ & {$[118]$} \\
\hline & ${ }^{106} \mathrm{Cd}$ & 1749 & $>4.1 \times 10^{20}$ & {$[117]$} \\
\hline & ${ }^{92} \mathrm{Mo}$ & 627.1 & $>1.9 \times 10^{20}$ & {$[119]$} \\
\hline & ${ }^{78} \mathrm{Kr}$ & 1844 & $>7 \times 10^{19}$ & {$[116]$} \\
\hline \multirow[t]{3}{*}{$2 \beta^{+}(0 \nu)$} & ${ }^{130} \mathrm{Ba}$ & 567 & $>4 \times 10^{21}$ & {$[112]$} \\
\hline & ${ }^{78} \mathrm{Kr}$ & 822 & $>1 \times 10^{21}$ & {$[116]$} \\
\hline & ${ }^{106} \mathrm{Cd}$ & 727 & $>2.4 \times 10^{20}$ & {$[117]$} \\
\hline \multirow[t]{3}{*}{$2 \beta^{+}(2 \nu)$} & ${ }^{130} \mathrm{Ba}$ & 567 & $>4 \times 10^{21}$ & {$[112]$} \\
\hline & ${ }^{78} \mathrm{Kr}$ & 822 & $>1 \times 10^{21}$ & {$[116]$} \\
\hline & ${ }^{106} \mathrm{Cd}$ & 727 & $>2.4 \times 10^{20}$ & {$[117]$} \\
\hline
\end{tabular}


Table 11. Best present limits on $\operatorname{ECEC}(0 \nu)$ to the excited state at a $90 \%$ C.L. for isotope-candidates with possible resonance enhancement. Here $\Delta M$ is the atomic mass difference of parent and daughter nuclei, $E^{*}\left(J^{\pi}\right)$ is the energy of the excited state of the daughter nuclide (with its spin and parity in parenthesis). ${ }^{*)}$ Extracted from results for the $\operatorname{ECEC}\left(2 \nu ; 0^{+}-0_{g . s .}^{+}\right)$transition obtained for ${ }^{78} \mathrm{Kr}[98]$ and for ${ }^{106} \mathrm{Cd}[99] ;{ }^{* *)}$ extracted from geochemical experiments [97, 112].

\begin{tabular}{lcccc}
\hline Nucleus Abundance, $\%$ & $\Delta \mathrm{M}, \mathrm{keV}$ & $E^{*}\left(J^{\pi}\right)$ & $T_{1 / 2}, \mathrm{y}$ \\
\hline${ }^{74} \mathrm{Se}$ & 0.89 & $1209.7 \pm 2.3$ & $1204.2\left(2^{+}\right)$ & $>5.5 \times 10^{18}[115]$ \\
${ }^{78} \mathrm{Kr}$ & 0.35 & $2846.4 \pm 2.0$ & $2838.9\left(2^{+}\right)$ & $>1 \times 10^{21 *)}$ \\
${ }^{96} \mathrm{Ru}$ & 5.52 & $2718.5 \pm 8.2$ & $2700(?)$ & - \\
${ }^{106} \mathrm{Cd}$ & 1.25 & $2770 \pm 7.2$ & $2741.0\left(1,2^{+}\right)$ & $\left.>1.5 \times 10^{20 *}\right)$ \\
& & & & $>3 \times 10^{19}[117]$ \\
${ }^{112} \mathrm{Sn}$ & 0.97 & $1919.5 \pm 4.8$ & $1871.0\left(0^{+}\right)$ & $>0.92 \times 10^{20}[105]$ \\
${ }^{130} \mathrm{Ba}$ & 0.11 & $2617.1 \pm 2.0$ & $2608.4(?)$ & $\left.>1.5 \times 10^{21 * *}\right)$ \\
${ }^{136} \mathrm{Ce}$ & 0.20 & $2418.9 \pm 13$ & $2399.9\left(1^{+}, 2^{+}\right)$ & - \\
& & & $2392.1\left(1^{+}, 2^{+}\right)$ & - \\
${ }^{162} \mathrm{Er}$ & 0.14 & $1843.8 \pm 5.6$ & $1745.7\left(1^{+}\right)$ & - \\
\hline
\end{tabular}

2002, the NEMO-3 detector has operated in the Frejus Underground Laboratory (France) located at a depth of $4800 \mathrm{~m}$ w.e. The detector has a cylindrical structure and consists of 20 identical sectors (see Fig.3). A thin $\left(30-60 \mathrm{mg} / \mathrm{cm}^{2}\right)$ source containing double beta-decaying nuclei and natural material foils have a total area of $20 \mathrm{~m}^{2}$ and a weight of up to $10 \mathrm{~kg}$ was placed in the detector. The basic principles of detection are identical to those used in the NEMO-2 detector. The energy of the electrons is measured by plastic scintillators (1940 individual counters), while the tracks are reconstructed on the basis of information obtained in the planes of Geiger cells (6180 cells) surrounding the source on both sides. The tracking volume of the detector is filled with a mixture consisting of $\sim 95 \% \mathrm{He}, 4 \%$ alcohol, $1 \% \mathrm{Ar}$ and $0.1 \%$ water at slightly above atmospheric pressure. In addition, a magnetic field with a strength of $25 \mathrm{G}$ parallel to the detector's axis is created by a solenoid surrounding the detector. The magnetic field is used to identify electron-positron pairs so as to suppress this source of background. 


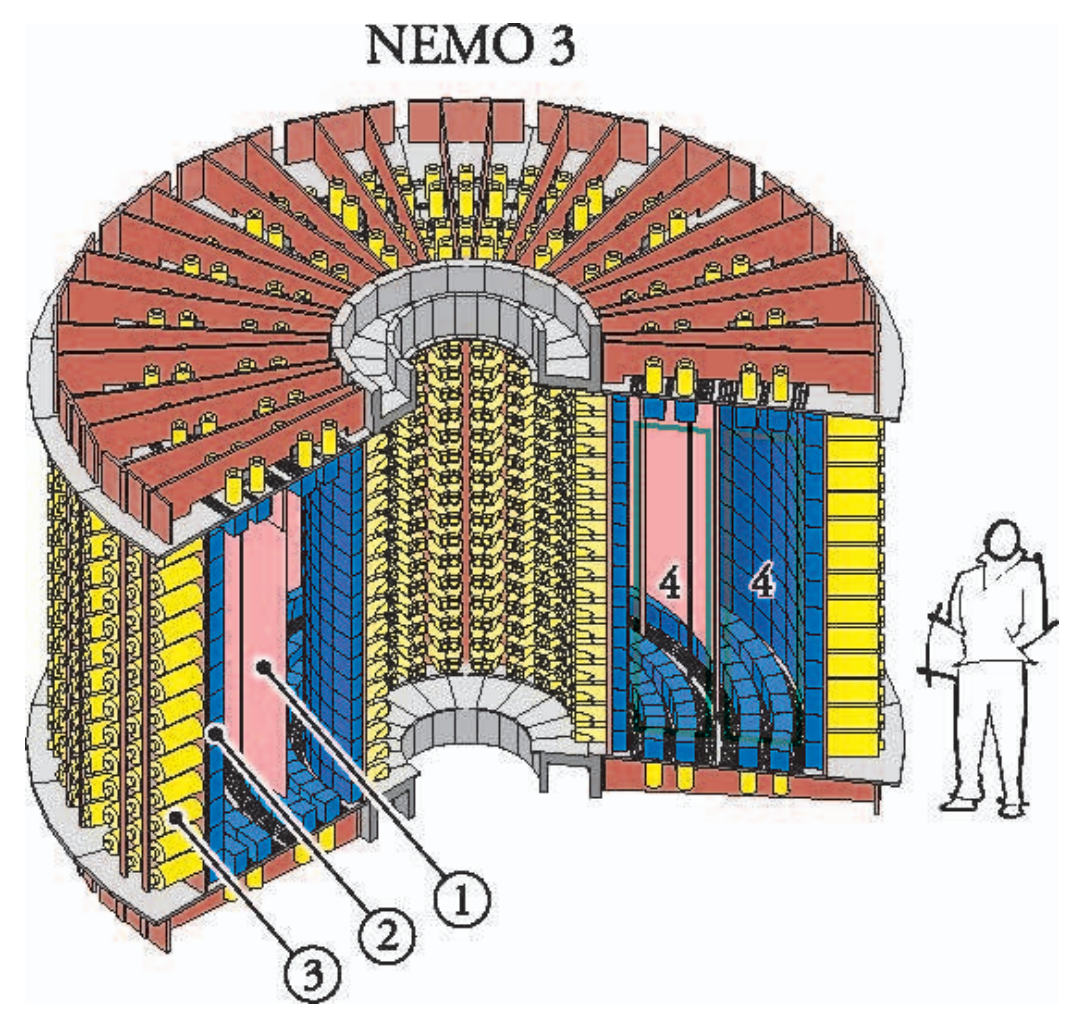

Figure 3. The NEMO-3 detector without shielding [120]. 1 - source foil; 2 - plastic scintillator; 3 low radioactivity PMT; 4 - tracking chamber.

The main characteristics of the detector are the following. The energy resolution of the scintillation counters lies in the interval 14-17\% FWHM for electrons of energy $1 \mathrm{MeV}$. The time resolution is $250 \mathrm{ps}$ for an electron energy of $1 \mathrm{MeV}$ and the accuracy in reconstructing the vertex of $2 \mathrm{e}^{-}$events is $1 \mathrm{~cm}$. The detector is surrounded by a passive shield consisting of $20 \mathrm{~cm}$ of steel and $30 \mathrm{~cm}$ of borated water. The level of radioactive impurities in structural materials of the detector and of the passive shield was tested in measurements with lowbackground HPGe detectors.

Measurements with the NEMO-3 detector revealed that tracking information, combined with time and energy measurements, makes it possible to suppress the background efficiently. That NEMO-3 can be used to investigate almost all isotopes of interest is a distinctive feature of this facility. At the present time, such investigations are being performed for seven isotopes; these are ${ }^{100} \mathrm{Mo},{ }^{82} \mathrm{Se},{ }^{116} \mathrm{Cd},{ }^{150} \mathrm{Nd},{ }^{96} \mathrm{Zr},{ }^{130} \mathrm{Te}$, and ${ }^{48} \mathrm{Ca}$ (see Table 12). As mentioned above, foils of copper and natural (not enriched) tellurium were placed in the detector to perform background measurements.

Fig. 4 and Fig. 5 display the spectrum of $2 \nu \beta \beta$ events for ${ }^{100} \mathrm{Mo}$ and ${ }^{82} \mathrm{Se}$ that were 
Table 12. Investigated isotopes with NEMO-3 [120].

\begin{tabular}{ccccccccc}
\hline Isotope & ${ }^{100} \mathrm{Mo}{ }^{82} \mathrm{Se}$ & ${ }^{130} \mathrm{Te}$ & ${ }^{116} \mathrm{Cd}$ & ${ }^{150} \mathrm{Nd}$ & ${ }^{96} \mathrm{Zr}$ & ${ }^{48} \mathrm{Ca}$ \\
\hline $\begin{array}{c}\text { Enrichment, } \\
\%\end{array}$ & 97 & 89 & 93 & 91 & 57 & 73 \\
Mass of & 6914 & 932 & 454 & 405 & 36.6 & 9.4 & 7.0 \\
isotope, $\mathrm{g}$ & & & & & & & \\
\hline
\end{tabular}
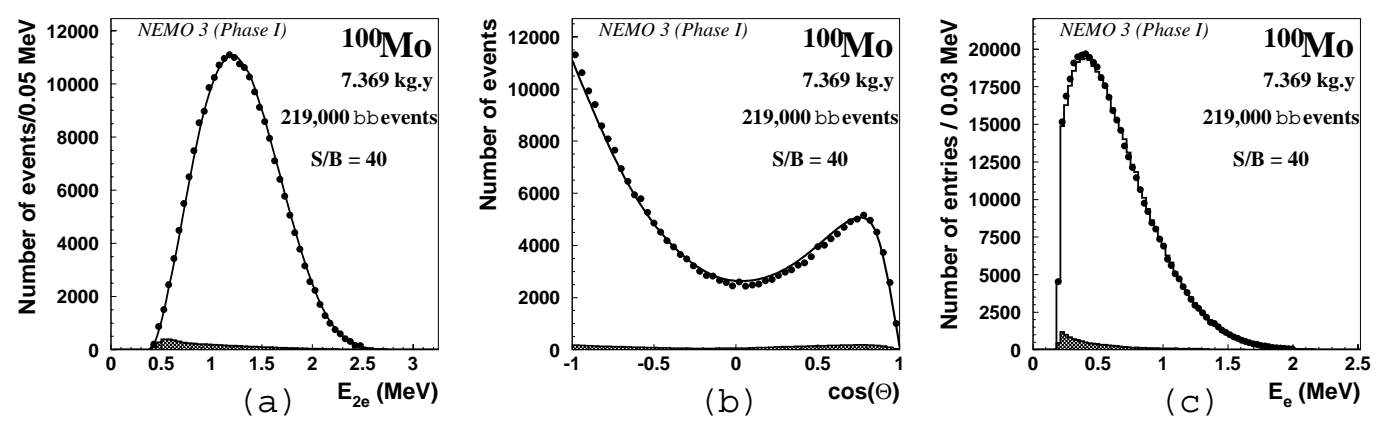

Figure 4. (a) Energy sum spectrum of the two electrons, (b) angular distribution of the two electrons and (c) single energy spectrum of the electrons, after background subtraction from ${ }^{100} \mathrm{Mo}$ with of $7.369 \mathrm{~kg} \cdot y e a r s$ exposure [47]. The solid line corresponds to the expected spectrum from $2 \nu \beta \beta$ simulations and the shaded histogram is the subtracted background computed by Monte-Carlo simulations.

collected over 389 days (Phase I) [47]. For ${ }^{100}$ Mo the angular distribution (Fig. 4b) and single electron spectrum (Fig. 4c) are also shown. The total number of events exceeds 219,000 which is much greater than the total statistics of all of the preceding experiments with ${ }^{100} \mathrm{Mo}$ (and even greater than the total statistics of all previous $2 \nu \beta \beta$ decay experiments!). It should also be noted that the background is as low as $2.5 \%$ of the total number of $2 \nu \beta \beta$ events. Employing the calculated values of the detection efficiencies for $2 \nu \beta \beta$ events, the following half-life values were obtained for ${ }^{100} \mathrm{Mo}$ and ${ }^{82} \mathrm{Se}$ [47]:

$$
T_{1 / 2}\left({ }^{100} M o ; 2 \nu\right)=[7.11 \pm 0.02(\text { stat }) \pm 0.54(\text { syst })] \cdot 10^{18} y
$$




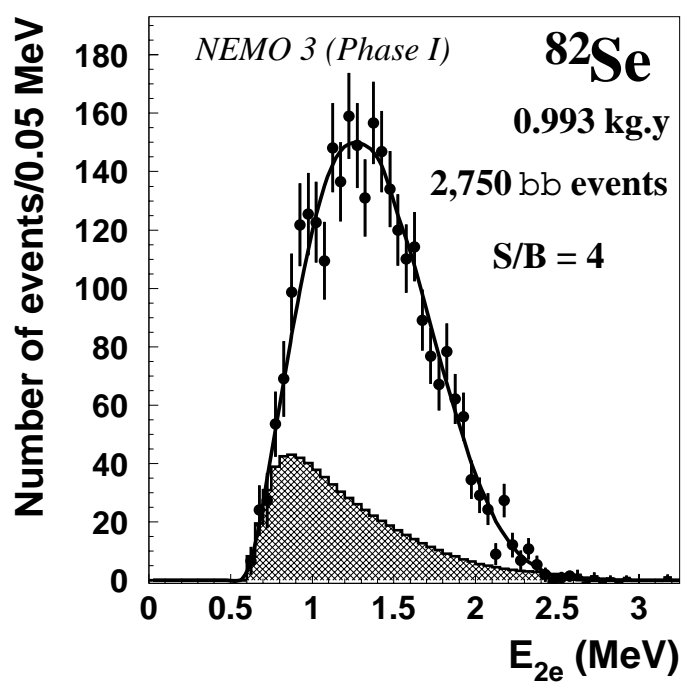

Figure 5. Energy sum spectrum of the two electrons after background subtraction from ${ }^{82} \mathrm{Se}$ with $0.993 \mathrm{~kg} \cdot y e a r s$ exposure (same legend as Fig. 4) [47]. The signal contains 2,750 $2 \beta$ events and the signal-to-background ratio is 4 .

$$
T_{1 / 2}\left({ }^{82} \mathrm{Se} ; 2 \nu\right)=[9.6 \pm 0.3(\text { stat }) \pm 1.0(\text { syst })] \cdot 10^{19} y
$$

These results and results for ${ }^{48} \mathrm{Ca},{ }^{96} \mathrm{Zr},{ }^{116} \mathrm{Cd},{ }^{130} \mathrm{Te}$ and ${ }^{150} \mathrm{Nd}$ are presented in Table 13. Notice that the values for ${ }^{100} \mathrm{Mo}$ and ${ }^{116} \mathrm{Cd}$ have been obtained on the assumption that the single state dominance (SSD) mechanism is valid ${ }^{5}[108,124]$. Systematic uncertainties can be decreased using calibrations and can be improved by up to $\sim(3-5) \%$.

Fig. 6 shows the tail of the two-electron energy sum spectrum in the $0 \nu \beta \beta$ energy window for ${ }^{100} \mathrm{Mo}$ and ${ }^{82} \mathrm{Se}$ (Phase I+II; 690 days of measurement). One can see that the experimental spectrum is in good agreement with the calculated spectrum, which was obtained taking into account all sources of background. Using a maximum likelihood method, the following limits on neutrinoless double beta decay of ${ }^{100} \mathrm{Mo}$ and ${ }^{82} \mathrm{Se}$ (mass mechanism; $90 \%$ C.L.) have been obtained:

$$
T_{1 / 2}\left({ }^{100} M o ; 0 \nu\right)>5.8 \cdot 10^{23} y
$$

${ }^{5}$ Validity of SSD mechanism in ${ }^{100}$ Mo was demonstrated using analysis of the single electron spectrum (see $[121,122])$. In the case of ${ }^{116} \mathrm{Cd}$ this is still a hypothesis. 
Table 13. Two neutrino half-life values for different nuclei obtained in the NEMO-3 experiment (for ${ }^{116} \mathrm{Cd},{ }^{96} \mathrm{Zr}{ }^{150} \mathrm{Nd},{ }^{48} \mathrm{Ca}$ and ${ }^{130} \mathrm{Te}$ the results are preliminary). First error is statistical and second is systematic; $\mathrm{S} / \mathrm{B}$ is the signal-to- background ratio.

\begin{tabular}{lcccc}
\hline Isotope & $\begin{array}{c}\text { Measurement } \\
\text { time, days }\end{array}$ & $\begin{array}{c}\text { Number of } \\
2 \nu \text { events }\end{array}$ & $\mathrm{S} / \mathrm{B}$ & $T_{1 / 2}(2 \nu), \mathrm{y}$ \\
\hline${ }^{100} \mathrm{Mo}$ & 389 & 219000 & 40 & $(7.11 \pm 0.02 \pm 0.54) \cdot 10^{18}[47]$ \\
${ }^{82} \mathrm{Se}$ & 2750 & 4 & $(9.6 \pm 0.3 \pm 1.0) \cdot 10^{19}[47]$ \\
${ }^{116} \mathrm{Cd}$ & 168 & 1371 & 7.5 & $(2.8 \pm 0.1 \pm 0.3) \cdot 10^{19}$ \\
${ }^{96} \mathrm{Zr}$ & 924.67 & 331 & 1 & $(2.3 \pm 0.2 \pm 0.3) \cdot 10^{19}$ \\
${ }^{150} \mathrm{Nd}$ & 939 & 2018 & 2.8 & $\left(9.2_{-0.22}^{+0.25} \pm 0.62\right) \cdot 10^{18}$ \\
${ }^{48} \mathrm{Ca}$ & 943.16 & 116 & 6.8 & $\left(4.4_{-0.40}^{+0.5} \pm 0.4\right) \cdot 10^{19}$ \\
${ }^{130} \mathrm{Te}$ & 534 & 109 & 0.2 & $(7.6 \pm 1.5 \pm 0.8) \cdot 10^{20}$ \\
\hline
\end{tabular}

$$
T_{1 / 2}\left({ }^{82} S e ; 0 \nu\right)>2.1 \cdot 10^{23} y
$$

Additionally, using NME values from [10-13] the bound on $\left\langle m_{\nu}\right\rangle$ gives $0.61-1.26 \mathrm{eV}$ for ${ }^{100} \mathrm{Mo}$ and $1.16-2.11 \mathrm{eV}$ for ${ }^{82} \mathrm{Se}$.

In this experiment the best present limits on all possible modes of double beta decay with Majoron emission have been obtained too (see Tables 3 and 4).

For the first running period (Phase I) radon was the dominant background in $0 \nu \beta \beta$ decay energy region. It has been significantly reduced by a factor $\sim 6$ by a radon-tight tent enclosing the detector and a radon-trap facility in operation since December 2004 which has started a second running period (Phase II). After five years of data collection, the expected sensitivity at $90 \%$ C.L will be $T_{1 / 2}(0 \nu \beta \beta)>2 \times 10^{24}$ y for ${ }^{100} \mathrm{Mo}$ and $8 \times 10^{23} \mathrm{y}$ for ${ }^{82} \mathrm{Se}$, corresponding to $\left\langle m_{\nu}\right\rangle<0.3-0.7 \mathrm{eV}$ for ${ }^{100} \mathrm{Mo}$ and $\left\langle m_{\nu}\right\rangle<0.6-1.1 \mathrm{eV}$ for ${ }^{82} \mathrm{Se}$. At the same time the search for decays with Majoron emission with a record sensitivity and a precise investigation of $2 \nu \beta \beta$ decay in the seven above mentioned nuclei will continue. 

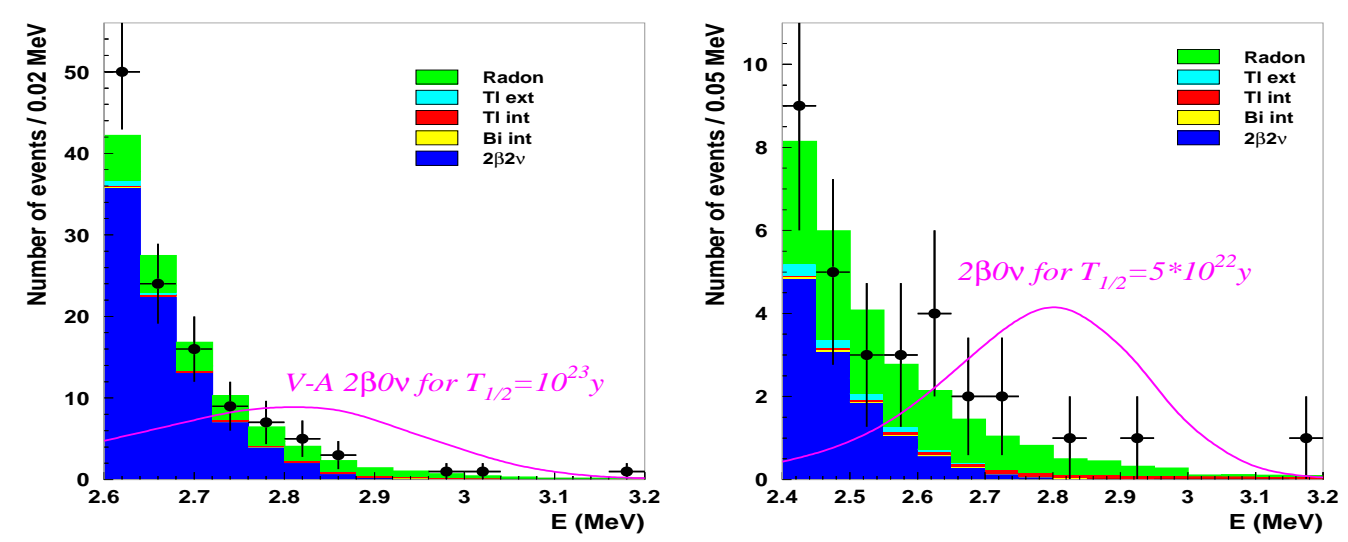

Figure 6. Distribution of the energy sum of two electrons for ${ }^{100} \mathrm{Mo}$ (left) and ${ }^{82} \mathrm{Se}$ (right) (Phase I + II data, 693 days of measurement).

\subsection{CUORICINO [45, 46$]$}

This program is the first stage of the larger CUORE experiment (see Subsection 4.1). The experiment is running at the Gran Sasso Underground Laboratory in Italy $(3500 \mathrm{~m}$ w.e.). The detector consists of low-temperature devices based on ${ }^{n a t} \mathrm{TeO}_{2}$ crystals. The use of natural tellurium is justified because the content of ${ }^{130} \mathrm{Te}$ in it is rather high, $33.8 \%$. The detector consists of 62 individual crystals, their total weight being $40.7 \mathrm{~kg}$. The energy resolution is $7.5-9.6 \mathrm{keV}$ at an energy of $2.6 \mathrm{MeV}$.

The experiment has been running since March of 2003. The summed spectra of all crystals in the region of the $0 \nu \beta \beta$ energy is shown in Fig. 7. The total exposure is $11.93 \mathrm{~kg} \cdot \mathrm{y}\left({ }^{130} \mathrm{Te}\right)$. The background at the energy of the $0 \nu \beta \beta$ decay is $0.18 \mathrm{keV}^{-1} \cdot \mathrm{kg}^{-1} \cdot \mathrm{y}^{-1}$. No peak is evident and the limit is $T_{1 / 2}>3 \cdot 10^{24}$ y $(90 \% \text { C.L. })^{6}$.

Using NME values from [10-13] the limit on $\left\langle m_{\nu}\right\rangle$ is less than $0.29-0.57 \mathrm{eV}$. If one uses the NME from Shell Model calculations [42] the $\left\langle m_{\nu}\right\rangle<0.75 \mathrm{eV}$.

The sensitivity of the experiment to $0 \nu \beta \beta$ decay of ${ }^{130}$ Te under the present conditions will be at the level of $\sim 5 \cdot 10^{24}(90 \%$ C.L.) for $5 \mathrm{y}$ of measurement. This in turn means the sensitivity to $\left\langle m_{\nu}\right\rangle$ is on the level of $0.22-0.44 \mathrm{eV}$. At the same time there is a hope to detect $2 \nu \beta \beta$ decay of ${ }^{130} \mathrm{Te}$.

\footnotetext{
${ }^{6}$ It should be stressed that "sensitivity" of the experiment under present conditions (when number of observed events is equal to expected mean background) is $\sim 2 \cdot 10^{24}$ y (90\% C.L.). Much better limit was obtained due to big "negative" fluctuation of the background in the $0 \nu$ energy region.
} 


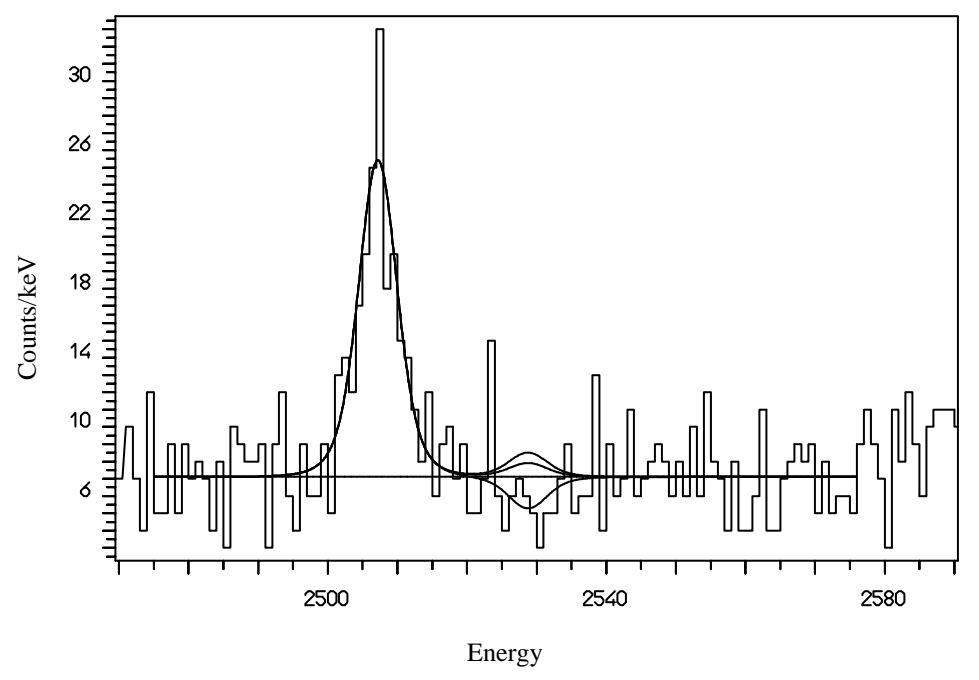

Figure 7. The sum spectra of all crystal in the region of the $0 \nu \beta \beta$ energy [46]. Energy is presented in $\mathrm{keV}$.

One of the tasks of the CUORICINO experiment is to demonstrate the possibility of substantially reducing the background to the level of $0.01-0.001 \mathrm{keV}^{-1} \cdot \mathrm{kg}^{-1} \cdot \mathrm{y}^{-1}$ which is necessary to proceed with the CUORE project (see section 4.1).

\section{PLANNED EXPERIMENTS}

Here five of the most developed and promising experiments which can be realized within the next five to ten years are discussed (see Table 14). The estimation of the sensitivity in the experiments is made using NMEs from [10-13, 42].

\subsection{CUORE [46, 125]}

This experiment will be run at the Gran Sasso Underground Laboratory (Italy; $3500 \mathrm{~m}$ w.e.). The plan is to investigate $760 \mathrm{~kg}$ of ${ }^{n a t} \mathrm{TeO}_{2}$, with a total of $\sim 200 \mathrm{~kg}$ of ${ }^{130} \mathrm{Te}$. One thousand low-temperature $(\sim 8 \mathrm{mK})$ detectors, each having a weight of $750 \mathrm{~g}$, will be manufactured and arranged in 19 towers. One tower is approximately equivalent to the CUORICINO detector, see Subsection 3.2. Planed energy resolution is 5 kev (FWHM). 
Table 14. Five most developed and promising projects (see text). Sensitivity at 90\% C.L. for three (1-st step of GERDA and MAJORANA) five (EXO, SuperNEMO and CUORE) and ten (full-scale GERDA and MAJORANA) years of measurements is presented. ${ }^{*)}$ For the background $0.001 \mathrm{keV}^{-1} \cdot \mathrm{kg}^{-1} \cdot \mathrm{y}^{-1}$; ${ }^{* *)}$ for the background $0.01 \mathrm{keV}^{-1} \cdot \mathrm{kg}^{-1} \cdot \mathrm{y}^{-1}$.

\begin{tabular}{cccccc}
\hline Experiment & Isotope & Mass of & Sensitivity & Sensitivity & Status \\
& & isotope, $\mathrm{kg}$ & $T_{1 / 2}, \mathrm{y}$ & $\left\langle m_{\nu}\right\rangle, \mathrm{meV}$ & \\
\hline CUORE [46, 125] & ${ }^{130} \mathrm{Te}$ & 200 & $6.5 \cdot 10^{26 *)}$ & $20-50$ & in progress \\
& & & $2.1 \cdot 10^{26 * *)}$ & $35-90$ & \\
GERDA [126] & ${ }^{76} \mathrm{Ge}$ & 40 & $2 \cdot 10^{26}$ & $70-300$ & in progress \\
& & 1000 & $6 \cdot 10^{27}$ & $10-40$ & R\&D \\
MAJORANA & ${ }^{76} \mathrm{Ge}$ & $30-60$ & $(1-2) \cdot 10^{26}$ & $70-300$ & R\&D \\
[129, 130] & & 1000 & $6 \cdot 10^{27}$ & $10-40$ & R\&D \\
EXO [131] & ${ }^{136} \mathrm{Xe}$ & 200 & $6.4 \cdot 10^{25}$ & $95-220$ & in progress \\
& & 1000 & $8 \cdot 10^{26}$ & $27-63$ & R\&D \\
SuperNEMO & ${ }^{82} \mathrm{Se}$ & $100-200$ & $(1-2) \cdot 10^{26}$ & $40-100$ & R\&D \\
[133-135] & & & & & \\
\hline
\end{tabular}

One of the problems here is to reduce the background level by a factor of about 10 to 100 in relation to the background level achieved in the detector CUORICINO [45, 46]. Upon reaching a background level of $0.001 \mathrm{keV}^{-1} \cdot \mathrm{kg}^{-1} \cdot \mathrm{y}^{-1}$, the sensitivity of the experiment to the $0 \nu$ decay of ${ }^{130}$ Te for 5 y of measurements and at $90 \%$ C.L. will become approximately $6.5 \cdot 10^{26} \mathrm{y}\left(\left\langle m_{\nu}\right\rangle \sim 0.02-0.05 \mathrm{eV}\right)$. For more realistic level of background $0.01 \mathrm{keV}^{-1} \cdot \mathrm{kg}^{-1} \cdot \mathrm{y}^{-1}$ sensitivity will be $\sim 2.1 \cdot 10^{26}$ y for half-life and $\sim 0.04-0.09 \mathrm{eV}$ for the effective Majorana neutrino mass. The experiment has been approved and funded.

\subsection{GERDA [126]}

This is one of two planned experiments with ${ }^{76} \mathrm{Ge}$ (along with the MAJORANA experiment). The experiment is to be located in the Gran Sasso Underground Laboratory (3500 
$m$ w.e.). The proposal is based on ideas and approaches which were proposed for GENIUS [1] and the GEM [127] experiments. The plan is to place "naked" HPGe detectors in highly purified liquid argon (as passive and active shield). It minimizes the weight of construction material near the detectors and decreases the level of background. The liquid argon dewar is placed into a vessel of very pure water. The water plays a role of passive and active (Cherenkov radiation) shield.

The proposal involves three phases. In the first phase, the existing HPGe detectors $(\sim 15$ $\mathrm{kg}$ ), which previously were used in the Heidelberg-Moscow [43] and IGEX [44] experiments, will be utilized. In the second phase $\sim 40 \mathrm{~kg}$ of enriched Ge will be investigated. In the third phase the plan is to use $\sim 500-1000 \mathrm{~kg}$ of ${ }^{76} \mathrm{Ge}$.

The first phase, lasting one year, is to measure with a sensitivity of $3 \cdot 10^{25} \mathrm{y}$, that gives a possibility of checking the "positive" result of [35-37]. The sensitivity of the second phase (for three years of measurement) will be $\sim 2 \cdot 10^{26} \mathrm{y}$. This corresponds to a sensitivity for $\left\langle m_{\nu}\right\rangle$ at the level of $\sim 0.07-0.3 \mathrm{eV}$.

The first two phases have been approved and funded. Measurements will start in $~ 2009$ 2010. The results of this first step will play an important role in the decision to support the full scale experiment.

The project is very promising although it will be difficult to reach the desired level of background. One of the significant problems is ${ }^{222} \mathrm{Rn}$ in the liquid argon (see, for example, results of [128]).

\subsection{MAJORANA [129, 130]}

The MAJORANA facility will consist of $\sim 420$ sectioned HPGe detectors manufactured from enriched germanium (the degree of enrichment is about 87\%). The total mass of enriched germanium will be $1000 \mathrm{~kg}$. The facility is designed in such a way that it will consist of 20 individual supercryostats manufactured from low radioactive copper, each containing 21 HPGe detectors. The entire facility will be surrounded by a passive shield and will be located at an underground laboratory in Canada (or in the United States). Only the total energy deposition will be utilized in measuring the $0 \nu \beta \beta$ decay of ${ }^{76}$ Ge to the ground state of the daughter nucleus. The use of sectioned HPGe detectors, pulse shape analysis, anticoincidence, and low radioactivity structural materials will make it possible to reduce 
the background to a value below $3 \cdot 10^{-4} \mathrm{keV}^{-1} \cdot \mathrm{kg}^{-1} \cdot \mathrm{y}^{-1}$ and to reach a sensitivity of about $6 \cdot 10^{27} \mathrm{y}$ within ten years of measurements. The corresponding sensitivity to the effective mass of the Majorana neutrino is about 0.01 to $0.04 \mathrm{eV}$. The measurement of the $0 \nu \beta \beta$ decay of ${ }^{76} \mathrm{Ge}$ to the $0^{+}$excited state of the daughter nucleus will be performed by recording two cascade photons and two beta electrons. The planned sensitivity for this process is about $10^{27} \mathrm{y}$.

In the first step $\sim 30-60 \mathrm{~kg}$ of ${ }^{76} \mathrm{Ge}$ will be investigated. It is anticipated that the sensitivity to $0 \nu \beta \beta$ decay to the ground state of the daughter nuclei for 3 years of measurement will be $(1-2) \cdot 10^{26} \mathrm{y}$. It will reject or to confirm the "positive" result from [35-37]. Sensitivity to $\left\langle m_{\nu}\right\rangle$ will be $\sim 0.07-0.3 \mathrm{eV}$. During this time different methods and technical questions will be checked and possible background problems will be investigated. The first step of MAJORANA will start at 2009-2010.

\subsection{EXO [131]}

In this experiment the plan is to implement M. Moe's proposal of 1991 [132]. Specifically it is to record both ionization electrons and the $\mathrm{Ba}^{+}$ion originating from the double-betadecay process ${ }^{136} \mathrm{Xe}-{ }^{136} \mathrm{Ba}^{++}+2 \mathrm{e}^{-}$. In reference [131], it is proposed to operate with $1 \mathrm{t}$ of ${ }^{136}$ Xe. The actual technical implementation of the experiment has not yet been developed. One of the possible schemes is to fill a TPC with liquid enriched xenon. To avoid the background from the $2 \nu$ decay of ${ }^{136} \mathrm{Xe}$, the energy resolution of the detector must not be poorer than $3.8 \%$ (FWHM) at an energy of $2.5 \mathrm{MeV}$ (ionization and scintillation signals will be detected).

In the $0 \nu$ decay of ${ }^{136} \mathrm{Xe}$, the TPC will measure the energy of two electrons and the coordinates of the event to within a few millimeters. After that, using a special stick Ba ions will be removed from the liquid and then will be registered in a special cell by resonance excitation. For $\mathrm{Ba}^{++}$to undergo a transition to a state of $\mathrm{Ba}^{+}$, a special gas is added to xenon. The authors of the project assume that the background will be reduced to one event within five years of measurements. Given a $70 \%$ detection efficiency it will be possible to reach a sensitivity of about $8 \cdot 10^{26} \mathrm{y}$ for the ${ }^{136} \mathrm{Xe}$ half-life and a sensitivity of about 0.03 to $0.06 \mathrm{eV}$ for the neutrino mass.

The authors also considered a detector in which the mass of ${ }^{136} \mathrm{Xe}$ is $10 \mathrm{t}$, but this is 
probably beyond present-day capabilities. It should be noted that about $100 \mathrm{t}$ of natural xenon are required to obtain 10 t of ${ }^{136} \mathrm{Xe}$. This exceeds the xenon produced worldwide over several years.

One should note that the principle difficulty in this experiment is associated with detecting the $\mathrm{Ba}^{+}$ion with a reasonably high efficiency. This issue calls for thorough experimental tests, and positive results have yet to be obtained.

As the first stage of the experiment EXO-200 will use $200 \mathrm{~kg}$ of ${ }^{136} \mathrm{Xe}$ without Ba ion identification. This experiment is currently under preparation and measurements will start probably in 2008-2009. The $200 \mathrm{~kg}$ of enriched Xe is a product of Russia with an enrichment of $\sim 80 \%$. If the background is 40 events in $5 \mathrm{y}$ of measurements, as estimated by the authors, then the sensitivity of the experiment will be $\sim 6 \cdot 10^{25} \mathrm{y}$. This corresponds to sensitivity for $\left\langle m_{\nu}\right\rangle$ at the level $\sim 0.1-0.2 \mathrm{eV}$. This initial prototype will operate at the Waste Isolation Pilot Plant (WIPP) in Southern New Mexico (USA).

\subsection{SuperNEMO [133-135]}

The NEMO Collaboration has studied and is pursing an experiment that will observe $100-200 \mathrm{~kg}$ of ${ }^{82} \mathrm{Se}$ with the aim of reaching a sensitivity for the $0 \nu$ decay mode at the level of $T_{1 / 2} \sim(1-2) \cdot 10^{26} \mathrm{y}$. The corresponding sensitivity to the neutrino mass is 0.04 to $0.1 \mathrm{eV}$. In order to accomplish this goal, it is proposed to use the experimental procedures nearly identical to that in the NEMO-3 experiment (see Subsection 3.1). The new detector will have planar geometry and will consist of 20 identical modules $\left(5 \mathrm{~kg}\right.$ of ${ }^{82} \mathrm{Se}$ in each sector). A ${ }^{82}$ Se source having a thickness of about $40 \mathrm{mg} / \mathrm{cm}^{2}$ and a very low content of radioactive admixtures is placed at the center of the modules. The detector will again record all features of double beta decay: the electron energy will be recorded by counters based on plastic scintillators $(\Delta E / E \sim 8-10 \%(F W H M)$ at $\mathrm{E}=1 \mathrm{MeV})$, while tracks will be reconstructed with the aid of Geiger counters. The same device can be used to investigate ${ }^{150} \mathrm{Nd},{ }^{100} \mathrm{Mo},{ }^{116} \mathrm{Cd}$, and ${ }^{130} \mathrm{Te}$ with a sensitivity to $0 \nu \beta \beta$ decay at a level of about $(0.5-1)$. $10^{26} \mathrm{y}$.

The use of an already tested experimental technique is an appealing feature of this experiment. The plan is to arrange the equipment at the new Frejus Underground Laboratory (France; the respective depth being $4800 \mathrm{~m}$ w.e.) or at CANFRANC Underground Labora- 
tory (Spain; 2500 m w.e.). The experiment is currently in its R\&D stage.

\section{CONCLUSIONS}

In conclusion, two-neutrino double-beta decay has so far been recorded for ten nuclei $\left({ }^{48} \mathrm{Ca},{ }^{76} \mathrm{Ge},{ }^{82} \mathrm{Se},{ }^{96} \mathrm{Zr},{ }^{100} \mathrm{Mo},{ }^{116} \mathrm{Cd},{ }^{128} \mathrm{Te},{ }^{130} \mathrm{Te},{ }^{150} \mathrm{Nd},{ }^{238} \mathrm{U}\right)$. In addition, the $2 \beta(2 \nu)$ decay of ${ }^{100} \mathrm{Mo}$ and ${ }^{150} \mathrm{Nd}$ to the $0^{+}$excited state of the daughter nucleus has been observed and the $\operatorname{ECEC}(2 \nu)$ process in ${ }^{130} \mathrm{Ba}$ was observed. Experiments studying two-neutrino double beta decay are presently approaching a qualitatively new level, where high-precision measurements are performed not only for half-lives but also for all other parameters of the process. As a result, a trend is emerging toward thoroughly investigating all aspects of two-neutrino double-beta decay, and this will furnish very important information about the values of NME, the parameters of various theoretical models, and so on. In this connection, one may expect advances in the calculation of NME and in the understanding of the nuclear physics aspects of double beta decay.

Neutrinoless double beta decay has not yet been confirmed. There is a conservative limit on the effective value of the Majorana neutrino mass at the level of $0.75 \mathrm{eV}$. Within the next few years, the sensitivity to the neutrino mass in the CUORICINO and NEMO-3 experiments will be improved to become about 0.2 to $0.5 \mathrm{eV}$ with measurements of ${ }^{130} \mathrm{Te}$ and ${ }^{100} \mathrm{Mo}$. It is precisely these two experiments that will carry out the investigations of double beta decay over the next few years. The next-generation experiments, where the mass of the isotopes being studied will be as grand as 100 to $1000 \mathrm{~kg}$, will have started within five to ten years. In all probability, they will make it possible to reach the sensitivity for the neutrino mass at a level of 0.01 to $0.1 \mathrm{eV}$.

1. H.V. Klapdor-Kleingrothaus, J. Hellmig and M. Hirsch, J. Phys. G 24, 483 (1998).

2. A. Faessler, F. Simkovic, Prog. Part. Nucl. Phys. 46, 233 (2001).

3. J.D.Vergados, Phys. Rep. 361, 1 (2002).

4. J.W.F. Valle, hep-ph/0608101.

5. S.M. Bilenky, J. Phys. A 40, 6707 (2007).

6. R.N. Mohapatra and A.Y. Smirnov, Ann. Rev. Nucl. Part. Sci. 56, 569 (2006). 
7. S. Pascoli, S.T. Petcov and W. Rodejohann, Phys. Lett. B 558, 141 (2003).

8. R.N. Mohapatra et al., hep-ph/0510213

9. S. Pascoli, S.T. Petcov and T. Schwetz, Nucl. Phys. B 734, 24 (2006).

10. V. Rodin et al., Nucl. Phys. A 766107 (2006); A 793, 213 (2007).

11. M. Kortelainen and J. Suhonen, Phys, Rev. C 75, 051203(R) (2007).

12. M. Kortelainen and J. Suhonen, Phys, Rev. C 76, 024315 (2007).

13. F. Simkovic et al., Phys, Rev. C 77, 045503 (2008).

14. A.S.Barabash, JETP Lett. 68, 1 (1998).

15. A.S. Barabash, Eur. Phys. J. A 8, 137 (2000).

16. A.S. Barabash, Astrophys. Space Sci. 283, 169 (2003).

17. A.D. Dolgov and A.Yu. Smirnov, Phys. Lett. B 621, 1 (2005).

18. A.S. Barabash et al., Nucl. Phys. B 783, 90 (2007).

19. G. Gelmini, M. Roncadelli, Phys. Lett. B 99, 1411 (1981).

20. C. Caso et al., (Particle Data Group) Eur. Phys. J. C 3, 1 (1998).

21. R.N. Mohapatra, P.B. Pal, Massive Neutrinos in Physics and Astrophysics, (World Scientific, Singapore, 1991).

22. Z.G. Berezhiani, A.Yu. Smirnov, J.W.F. Valle, Phys. Lett. B 291, 99 (1992).

23. R.N. Mohapatra, E. Takasugi, Phys. Lett. B 211, 192 (1988).

24. C.P. Burgess, J.M. Cline, Phys. Lett. B 298, 141 (1993); Phys. Rev. D 49, 5925 (1994).

25. P. Bamert, C.P. Burgess, R.N. Mohapatra, Nucl. Phys. B 449, 25 (1995).

26. C.D. Carone, Phys. Lett. B 308, 85 (1993).

27. R.N. Mohapatra, A. Perez-Lorenzana, C.A.S. Pires, Phys. Lett. B 491, 143 (2000).

28. V.I. Tretyak, Yu.G. Zdesenko, Atomic Data and Nuclear Data Tables 80, 83 (2002).

29. M.G. Inghram, J.H. Reynolds, Phys. Rev. 78, 822 (1950).

30. T. Kirsten, W. Gentner, O.A. Schaeffer, Z. Phys. 202, 273 (1967).

31. S.R. Elliott, A.A. Hahn, M.K. Moe, Phys. Rev. Lett. 59, 2020 (1987).

32. A.L. Turkevich, T.E. Economou and G.A. Cowan, Phys. Rev. Lett. 67, 3211 (1991).

33. A.S. Barabash, Czech. J. Phys. 56, 437 (2006); nucl-ex/0602009.

34. A.S. Barabash, AIP Conf. Proc. 942, 8 (2007); nucl-ex/0710.2194.

35. H.V. Klapdor-Kleingrothaus et al., Phys. Lett. B 586, 198 (2004).

36. H.V. Klapdor-Kleingrothaus et al., Mod. Phys. Lett. A 21, 1547 (2006). 
37. H.V. Klapdor-Kleingrothaus et al., Mod. Phys. Lett. A 16, 2409 (2001).

38. A.M. Bakalyarov et al., Phys. Part. Nucl. Lett. 2, 77 (2005); hep-ex/0309016.

39. C.E. Aalseth et al., Mod. Phys. Lett. A 17, 1475 (2002).

40. Yu.G. Zdesenko, F.A. Danevich and V.I. Treyak, Phys. Lett. B 546, 206 (2002).

41. A. Strumia and F. Vissani, Nucl. Phys. B 726, 294 (2005).

42. E. Caurier et al., Phys. Rev. Lett. 100, 052503 (2008).

43. H.V. Klapdor-Kleingrothaus et al., Eur. Phys. J. A 12, 147 (2001).

44. C.E. Aalseth et al., Phys. Rev. C 65, 09007 (2002).

45. C. Arnaboldi et al., Phys. Rev. Lett. 95, 142501 (2005).

46. C. Arnaboldi et al., hep-ex/0802.3439.

47. R. Arnold et al., Phys. Rev. Lett. 95, 182302 (2005).

48. R. Bernabei et a., Phys. Lett. B 546, 23 (2002).

49. F.A. Danevich et al., Phys. Rev. C 67, 035501 (2003).

50. R. Arnold et al., Nucl. Phys. A 765, 483 (2006).

51. O.K. Manuel, J. Phys. G 17, 221 (1991).

52. M. Gunther et al., Phys. Rev. D 55, 54 (1997).

53. R. Arnold et al., Nucl. Phys. A 678, 341 (2000).

54. J. Tanaka J., H. Ejiri, Phys. Rev. D 48, 5412 (1993).

55. C. Arnaboldi et al., Phys. Lett. B 557, 167 (2003).

56. E. Fiorini, in Proc. Int. Conf. NEUTRINO'r7 ("Nauka", Moscow, 1978), Vol. 2, p. 315.

57. E. Bellotti et al., Lett. Nuovo Chim. 33, 273 (1982).

58. A.S. Barabash, JETP Lett. 51, 207 (1990); Preprint 188-89, ITEP (Moscow, 1989).

59. A.S. Barabash et al., Phys. Lett. B 345, 408 (1995).

60. A.S. Barabash et al., Phys. At. Nucl. 62, 2039 (1999).

61. L. De Braeckeleer et al., Phys. Rev. Lett. 86, 3510 (2001).

62. M.J. Hornish et al., Phys. Rev. C 74, 044314 (2006).

63. R. Arnold et al., Nucl. Phys. A 781, 209 (2007).

64. A.S. Barabash et al., JETP Lett. 79, 10 (2004).

65. A.S. Barabash, Czech. J. Phys. 50, 447 (2000).

66. A.S. Barabash, Phys. At. Nucl. 67, 438 (2004).

67. A.A. Raduta and C.M. Raduta, Phys. Lett. B 647, 265 (2007). 
68. M. Aunola and J. Suhonen, Nucl. Phys. A 602, 133 (1996).

69. J. Toivanen and J. Suhonen, Phys. Rev. C 55, 2314 (1997).

70. A. Bakalyarov et al., JETP Lett. 76, 545 (2002).

71. C. Arpesella et al., Nucl. Phys. B (Proc. Suppl.) 70, 249 (1999).

72. A.S. Barabash et al., J.Phys. G 22, 487 (1996).

73. S. Stoica and I. Mihut, Nucl. Phys, A 602, 197 (1996).

74. J. Suhonen et al., Z. Phys. A 358, 297 (1997).

75. E. Bellotti et al., Europhys. Lett. 3, 889 (1987).

76. A. Piepke et al., Nucl. Phys. A 577, 493 (1994).

77. A.S. Barabash et al., Z. Phys. A 352, 231 (1995).

78. J.G. Hirsch et al., Phys. Rev. C 51, 2252 (1995).

79. A.A. Klimenko et al., Czech. J. Phys. 52, 589 (2002).

80. A.S. Barabash et al., Eur. Phys. J. A 11, 143 (2001).

81. T. Tomoda, Phys. Lett. B 474, 245 (2000).

82. B. Maier, Nucl. Phys. B (Proc. Suppl.) 35, 358 (1994).

83. E. Bellotti et al., J. Phys. G 17, S231 (1991).

84. R. Arnold et al., Nucl. Phys. A 636, 209 (1998).

85. J. Suhonen, Phys. Rev. C 62, 042501 (2001).

86. F. Simkovic and A. Faessler, Prog. Part. Nucl. Phys. 48, 201 (2002).

87. A. Morales et al., Nuovo Ciment, A 100, 525 (1988).

88. J. Suhonen, Phys. Lett. B 477, 99 (2000).

89. J. Suhonen, Nucl. Phys. A 700, 649 (2002).

90. J. Suhonen and M. Aunola, Nucl. Phys. A 723, 271 (2003).

91. F. Simkovic et al., Phys. Rev. C 64, 035501 (2001).

92. M. Hirsch et al., Z. Phys. A 347, 151 (1994).

93. M. Doi and T. Kotani, Prog. Theor. Phys. 89, 139 (1993).

94. Z. Sujkowski and S. Wycech, Phys. Rev. C 70, 052501(R) (2004).

95. D. Vergados, Nucl.Phys. B 218, 109 (1983).

96. A.S. Barabash, JETP Lett. 59, 644 (1994).

97. A. Meshik et al., Phys. Rev. C 64, 035205 (2001).

98. Ju.M. Gavriljuk et al., Phys. At. Nucl. 69, 2124 (2006). 
99. P. Benes et al., in AIP Conf. Proc. 942, 19 (2007).

100. A.S. Barabash et al., J. Phys. G 34, 1721 (2007).

101. J.R. Wilson, Czech. J. Phys. 56, 543 (2006).

102. A.S. Barabash et al., Nucl. Phys. A 785, 371 (2007).

103. P. Belli et al., Phys. Lett. B 658, 193 (2008).

104. H.J. Kim et al., Nucl. Phys. A 793, 171 (2007).

105. A.S. Barabash et al., Nucl. Phys. A 807 , 269 (2008); nucl-ex/0804.3849.

106. J. Dawson et al., Nucl. Phys. A 799, 167 (2008).

107. J. Bernabeu et al., Nucl. Phys. B 223, 15 (1983).

108. P. Domin et al., Nucl. Phys. A 753, 337 (2005).

109. R. Chandra et al., Eur. Phys. J. A 23, 235 (2005).

110. P.K. Raina et al., Eur. Phys. J. A 28, 27 (2006).

111. A. Shukla P.K. Raina and P.K. Rath, J. Phys. G 34, 549 (2007).

112. A.S. Barabash and R.R. Saakyan, Phys. At. Nucl. 59, 179 (1996).

113. R.G. Winter, Phys. Rev. 100, 142 (1955).

114. M. Voloshin, G. Mizelmacher and R. Eramzhan, JETP Lett. 35, 656 (1982).

115. A.S. Barabash et al., Nucl. Phys. A 785, 371 (2007).

116. C. Saenz et al., Phys. Rev. C 50, 1170 (1994).

117. P. Belli et al., Astropart. Phys. 10, 115 (1999).

118. S.I. Vasiliev et al., JETP Lett. 57, 320 (1993).

119. A.S. Barabash et al., Z. Phys. A 357, 351 (1997).

120. R. Arnold et al., Nucl. Instr. Meth. A 536, 79 (2005).

121. R. Arnold et al., JETP Lett. 80, 377 (2004).

122. Yu.A. Shitov, Phys. At. Nucl., bf 69, 2090 (2006).

123. R. Arnold et al., Nucl. Instr. Meth. A 354, 338 (1995).

124. F. Simkovic, P. Domin and S. Semenov, J. Phys. G 27, 2233 (2001).

125. C. Arnaboldi et al., Nucl. Instr. Meth. A 518, 775 (2004).

126. I. Abt et al., hep-ex/0404039.

127. Yu.G. Zdesenko, O.A. Ponkratenko, V.I. Tretyak, J. Phys. G 27, 2129 (2001).

128. H.V. Klapdor-Kleingrothaus et al., Nucl. Instr. Meth. A 530, 410 (2004).

129. Majorana White Paper, nucl-ex/0311013. 
130. C.E. Aalseth et al., Nucl. Phys. B (Proc. Suppl.) 138, 217 (2005).

131. M. Danilov et al., Phys. Lett. B 480, 12 (2000).

132. M. Moe, Phys. Rev. C 44, R931 (1991).

133. A.S. Barabash, Czech. J. Phys. 52, 575 (2002).

134. A.S. Barabash, Phys. At. Nucl. 67, 1984 (2004).

135. F. Piquemal, Phys. At. Nucl. 69, 2096 (2006). 